\title{
Pricing Games among Interconnected Microgrids
}

\author{
Gaurav S. Kasbekar and Saswati Sarkar
}

\begin{abstract}
We consider a scenario with multiple independent microgrids close to each other in a region that are connected to each other and to the central grid (macrogrid). In each time slot, a given microgrid may produce more than, less than or as much power as it needs, and there is uncertainty on which of these events may occur. The microgrids with excess power, those with deficit power and the macrogrid trade power in an electricity market, in which each microgrid with excess power quotes a price for it and the microgrids with deficit power buy power from the microgrids who quote the lowest prices. This results in price competition among the microgrids with excess power, and this competition has several distinguishing features not normally present in price competition in traditional markets studied in economics. We analyze this price competition using the framework of game theory, explicitly compute a Nash Equilibrium and show its uniqueness.
\end{abstract}

\section{INTRODUCTION}

Traditional power systems often generate power in large power stations using fossil fuel resources, and distribute it over long distances. This results in depletion of fossil fuel resources, environmental pollution and large energy losses during distribution [4]. Microgrids, which are emerging as a promising alternative to traditional centralized power systems, mitigate the above problems through distributed energy generation close to the loads and the use of renewable energy sources [3], [4].

Microgrids are small-scale power supply networks that are designed to supply electric power to small communities such as housing complexes, universities, schools, industrial estates etc. [4]. A microgrid consists of an interconnected network of several energy sources such as solar panels, wind power stations, fuel cells and microturbines, and electrical loads such as households and factories [4]. The energy sources, called microsources, are typically of smaller capacity than the large generators in traditional power systems and are located close to the loads [4].

A microgrid is connected to the central power grid, called macrogrid, and to other microgrids in its vicinity, and can transfer power to or receive power from them. Now, there is often uncertainty in the amount of power generated by a given microsource in a microgrid and also in the amount of power consumed by a given load, e.g., the amount of power generated by a solar panel depends on the weather, and the amount of power consumed by a household depends on the electrical equipment that is being used- both of the above factors are uncertain. So, in a given time slot, the aggregate power generated by all the microsources within a microgrid may be more than, less than or equal to the aggregate power consumed by all the loads in the microgrid. Thus, in each slot, a microgrid generates more than, less than or as much aggregate power as it requires.

G. Kasbekar and S. Sarkar are with the Department of Electrical and Systems Engineering at University of Pennsylvania, Philadelphia, PA, U.S.A. Their email addresses are kgaurav@seas.upenn.edu and swati@seas.upenn.edu respectively.
Electricity deficits in a microgrid must be compensated through electricity trades with the macrogrid and also with other microgrids. Note that trades among neighboring microgrids may incur lower costs than trades of the microgrids with the macrogrid due to elimination of electricity wastage during long distance transmission. In fact, the above trade may be viewed as a potential opportunity, and "the bigger promise of microgrids may be in the private sector, not as islands of power unto themselves, but as trading partners, making and sharing electricity with each other and the grid at large [14]." Although significant progress has been made in the microgrid generation technology, electricity trades among microgrids have received limited attention [15]. The existing schemes are primarily centralized [16] and mostly auction-based (requiring a centralized auctioneer) [17]. Note that centralized schemes are not likely to scale as the technology proliferates. The penetration of this nascent technology is therefore contingent on the design of a distributed and scalable electricity trading framework equipped with dynamic pricing strategies that adapt to the load and price fluctuations of the macrogrid and all the microgrids. This design may not be accomplished adopting the macro-economics viewpoint that has been extensively used for analyzing traditional electricity markets (even those that consider renewable electricity generations) e.g., [18], [19], [20]: a macro-economic market consists of an infinite number of buyers and sellers and the competition is perfect in that the price of the commodity to be traded is exogenous and not affected by the choices made by the constituents of the market, but may be determined so as to maximize a social utility e.g., [21], or based on risk neutral pricing (which assumes arbitrary storage capabilities) e.g., [22], [23], [24], [25], [26]. Given that the microgrid technology is now growing (albeit rapidly as projected), starting from the stage that microgrids have only been experimentally deployed so far, a microeconomic analysis that considers a market with a finite number of entities and allows the trade dynamics to depend on the choices of and the uncertainties experienced by the individual entities is imperative. This is the space where this paper seeks to contribute.

We consider the following mechanism for the trade of power among neighboring microgrids in a region and the macrogrid: at the beginning of every time slot, each microgrid that expects to have excess power in the slot announces a price at which it is willing to sell the power. The microgrids who expect to have deficit power then buy power from the microgrids with excess power who set the lowest prices (and from the macrogrid if the total amount of power required by the microgrids with deficit power is not available with the microgrids with excess power). This results in price competition among the microgrids with excess power. If a microgrid quotes a low price, it will attract buyers, but will earn lower profit per sale. This is a common feature of an oligopoly [1], in which multiple firms sell a common good to a pool of buyers. Price competition in an oligopoly is naturally modeled using game theory [2], and 
has been extensively studied in economics using, for example, the classic Bertrand game [1], [8] and its variants.

However, a microgrid market has several distinguishing features, which makes the price competition very different from oligopolies encountered in economics. First, in every slot, each microgrid may have excess power, deficit power or neither. Thus, each microgrid who has excess power is uncertain about the number of microgrids from whom it will face competition as well as the demand for power. A low price will result in unnecessarily low revenues in the event that very few other microgrids have excess power or several microgrids have deficit power, because even with a higher price the microgrid's power would have been bought, and vice versa. Second, note that the sets of buyers and sellers in a microgrid market are drawn from the same pool of traders (the set of all the microgrids in the vicinity and the macrogrid), whereas in most traditional markets, the sets of buyers and sellers are distinct.

In this paper, we analyze price competition among interconnected microgrids in a region using the framework of game theory [2] and study Nash Equilibria (NE) [2] in the game. We model the system by assuming that in each slot, a microgrid may either have one unit of excess power, one unit of deficit power or may have neither excess nor deficit power, with some probabilities (Section II). First, in Section III, we consider the case where the price at which the macrogrid sells unit power is constant and known to the microgrids, and each microgrid has deficit power with the same probability, although the probability that a microgrid has excess power may be different for different microgrids. Since prices can take real values, the strategy sets of the microgrids are continuous. In addition, the utilities of the microgrids are not continuous functions of their actions. Thus, classical results, including those for concave and potential games, do not establish the existence and uniqueness of $\mathrm{NE}$ in the resulting game, and there is no standard algorithm for finding a NE. Nevertheless, we are able to explicitly compute a NE and show its uniqueness, allowing for player strategies that are arbitrary mixtures of continuous and discrete probability distributions. The structure of the NE reveals several interesting insights, which we discuss in Section III-C. Next, in Section IV, we consider the model analyzed in Section III with arbitrary deficit power probabilities of the microgrids and explicitly compute a NE for the case of three microgrids. Then, in Section V, we generalize the results in Section III to the case where the price at which the macrogrid sells unit power is a random variable. We provide numerical studies in Section VI and conclude in Section VII.

In the economics literature, the Bertrand game [1], [8] and several of its variants have been used to study price competition. The closest to our work are [10], [11], which analyze price competition where each seller may be inactive with some probability. In our prior work [12], [13], we analyzed price competition among primary users in a Cognitive Radio Network- in that model, each primary may have unused bandwidth with some probability, which it can sell to a secondary user. However, [10], [11], [12] suffer from the limitation that they consider only the symmetric model where the good availability probability of each seller is the same ${ }^{1}$. Also, in all of the above papers [10], [11], [12], [13], the set of sellers and the set of buyers are distinct, whereas in case of price competition among interconnected microgrids, the sellers and buyers are drawn from the same set of traders.

The facts that in the game we consider, (i) there is uncertainty in whether a given trader (microgrid) has the good (excess power) to sell in a slot and (ii) the sets of sellers and buyers are drawn from the same pool of traders result in significant changes in the structure of the NE in comparison with that in games where one or both of the above features are not present. For example, in the Bertrand price competition game [8], which does not have features (i) and (ii), there is a unique NE, which is of pure strategy type [8]. On the other hand, in the game in this paper, no pure strategy NE exists and there is a unique NE, which is of mixed strategy type, provided there are at least three microgrids in the system. If there are two microgrids, there is a pure strategy NE in the game in this paper, but the NE strategies are different from those in the Bertrand game. Also, the NE in the game studied in [13] (see the preceding paragraph), which has feature (i) but not feature (ii) above, is of mixed-strategy type for the case of two sellers, and hence differs in structure from the game in this paper. Finally, in the game in this paper, for the case of three microgrids, when the probabilities of the microgrids having deficit power are asymmetric, the expected utilities that the microgrids get are also asymmetric, in contrast to the game in [13], in which the expected utilities are always equal.

The proofs of all the analytical results are relegated to the Appendix.

\section{MODEL}

We consider a scenario in which there are $n$ microgrids close to each other in a region. Each microgrid consists of an interconnected network of microsources (e.g., solar panels, fuel cells, wind power generators) and loads (e.g., households, factories, shops). Also, the $n$ microgrids are connected to each other and each of the $n$ microgrids is connected to the central grid or macrogrid.

In each microgrid, the microsources are capable of generating electrical power and each load has some demand for power. Time is divided into slots of equal duration. In each slot, there is uncertainty in the amount of power generated by a given microsource as well as the amount of power consumed by a given load. For example, the amount of power generated by a solar panel depends on the weather, and the amount of power consumed by a household depends on the weather, the time of the day, the electrical equipment that is being operated etc.all of the above factors are uncertain. So in each slot, a given microgrid either generates more, less or as much aggregate power as it requires. To model this, we assume that in every slot, each microgrid $i \in\{1, \ldots, n\}$ independently has 1 unit of excess power with probability (w.p.) $q_{i}, 1$ unit of deficit power w.p. $s_{i}$ and neither excess nor deficit w.p. $1-q_{i}-s_{i}$, where $q_{i}>0, s_{i}>0$ and $q_{i}+s_{i}<1$. Also, we assume that each microgrid knows whether it will have excess power, deficit power or neither in a slot at the beginning of the slot.

Each microgrid is capable of drawing power from or transferring power to the macrogrid or another microgrid. Since

\footnotetext{
${ }^{1}$ In [12], a toy model with 2 sellers and 1 buyer is analyzed in the case with asymmetric good availability probabilities of the sellers.
} 
microgrids as well as the macrogrid are selfish entities, the transfer of power by any of these entities is done in exchange for a fee. The macrogrid buys power from microgrids at the rate of $c$ per unit and sells power to microgrids at the rate of $v$ per unit, where we assume that $c<v$ since the macrogrid incurs some cost for transmission and distribution of power over long distances and also makes some profit. We assume that $c$ and $v$ are constant and known to all the microgrids, except in Section $\mathrm{V}$, in which we consider the case where $v$ is a random variable.

Now, each microgrid that has 1 unit of excess power announces a price $p_{i}$ at which it is willing to sell power to a microgrid that has 1 unit of deficit power. Note that a microgrid with excess power always has the option of selling its power to the macrogrid for a price of $c$; so $p_{i} \geq c$. Similarly, a microgrid with deficit power has the option of buying power from the macrogrid for a price of $v$; so when $v$ is constant and known, $p_{i} \leq v$.

Let $N$ (respectively, $K$ ) be the number of microgrids with excess power (respectively, deficit power) in a slot. If $K \leq N$, then the microgrids that have deficit power buy power from the microgrids offering the $K$ lowest prices among those who have excess power. If $K>N$, then $N$ of the microgrids with deficit power buy power from the microgrids with excess power and the remaining $K-N$ buy power from the macrogrid.

If microgrid $j$ sets ${ }^{2}$ a price $p_{j}, j=1, \ldots, n$, and microgrid $i$ has 1 unit of excess power, we define the utility $u_{i}\left(p_{1}, \ldots, p_{n}\right)$ of microgrid $i$ to be the incremental revenue that it earns over and above its revenue if it were to sell its power to the macrogrid; so $u_{i}\left(p_{1}, \ldots, p_{n}\right)=p_{i}-c$ if microgrid $i$ sells its power to another microgrid at price $p_{i}$, and $u_{i}\left(p_{1}, \ldots, p_{n}\right)=0$ if microgrid $i$ sells its power to the macrogrid for price $c$.

We allow each microgrid $i$ to choose its price $p_{i}$ randomly from a set of prices using an arbitrary distribution function ${ }^{3}$ (d.f.) $\psi_{i}($.$) , which is referred to as the strat-$ egy of microgrid $i$. The vector $\left(\psi_{1}(),. \ldots, \psi_{n}().\right)$ of strategies of the microgrids is called a strategy profile [1]. Let $\psi_{-i}=\left(\psi_{1}(),. \ldots, \psi_{i-1}(),. \psi_{i+1}(),. \ldots, \psi_{n}().\right)$ denote the vector of strategies of the microgrids other than $i$. Let $E\left\{u_{i}\left(\psi_{i}(),. \psi_{-i}\right)\right\}$ denote the expected utility of microgrid $i$ when it adopts strategy $\psi_{i}($.$) and the other microgrids adopt$ $\psi_{-i}$. If the strategy $\psi_{i}($.$) consists of setting the single price$ $p_{i}$ w.p. 1 , then we also denote the above expected utility by $E\left\{u_{i}\left(p_{i}, \psi_{-i}\right)\right\}$.

We use the Nash Equilibrium (NE) solution concept, which has been extensively used in game theory as a prediction of the outcome of a game. A NE is a strategy profile such that no player can improve his expected utility by unilaterally deviating from his strategy [1]. Thus, in our context, $\left(\psi_{1}^{*}(),. \ldots, \psi_{n}^{*}().\right)$ is a $\mathrm{NE}$ if for each microgrid $i: E\left\{u_{i}\left(\psi_{i}^{*}(),. \psi_{-i}^{*}\right)\right\} \geq E\left\{u_{i}\left(\tilde{\psi}_{i}(),. \psi_{-i}^{*}\right)\right\}, \forall \tilde{\psi}_{i}($.$) . When$ players other than $i$ play $\psi_{-i}^{*}, \psi_{i}^{*}($.$) maximizes i$ 's expected utility and is thus its best-response [1] to $\psi_{-i}$. Our goal is to find $\mathrm{NE}$ in the above price competition game and to investigate its uniqueness.

\footnotetext{
${ }^{2}$ If microgrid $j$ has no excess power, it does not matter what price $p_{j}$ it sets. Yet, for convenience, we speak of $p_{j}$ as being its action.

${ }^{3}$ Recall that the distribution function of a random variable (r.v.) $X$ is the function $G(x)=P(X \leq x)[5]$.
}

Now, if $n=2$, then there are only two microgrids, say 1 and 2 . There is no price competition between them, since in no event are they simultaneously prospective sellers to a common set of buyer microgrids, and it is easy to check that the strategy profile in which both microgrids $i=1,2$ set the price $p_{i}=v$ w.p. 1 is the unique NE. So henceforth, we assume that $n \geq 3$.

\section{Symmetric Deficit Power Probabilities}

For tractability, in the rest of this section, we assume that $s_{1}=\ldots=s_{n}=s$ for some $s \in(0,1)$. Note that $q_{1}, \ldots, q_{n}$ need not be equal. In Section IV, we analyze the generalization where $s_{1}, \ldots, s_{n}$ may be unequal (and $q_{1}, \ldots, q_{n}$ may also be unequal) for the case $n=3$.

Without loss of generality, we assume that $q_{1}, \ldots, q_{n}$ satisfy:

$$
q_{1} \geq q_{2} \geq \ldots \geq q_{n}
$$

For convenience, we define the pseudo-price of microgrid $i \in\{1, \ldots, n\}, p_{i}^{\prime}$, as the price it selects if it has excess power and $p_{i}^{\prime}=v+1$ otherwise ${ }^{4}$. Also, let $\phi_{i}($.$) be the d.f. of p_{i}^{\prime}$. For $c \leq x \leq v, p_{i}^{\prime} \leq x$ for a microgrid $i$ iff it has excess power and sets a price $p_{i} \leq x$. So $\phi_{i}(x)=q_{i} P\left(p_{i} \leq x\right)=q_{i} \psi_{i}(x)$. Thus, $\psi_{i}($.$) and \phi_{i}($.$) differ only by a constant factor on [c, v]$ and we use them interchangeably wherever applicable.

In Section III-A, we state some necessary conditions that any profile of NE strategies must satisfy. In Section III-B, we note that these conditions are sufficient and also explicitly compute the NE and show its uniqueness. In Section III-C, we discuss the insights that the structure of this NE provides.

\section{A. Necessary Conditions for a NE}

Consider a NE under which the d.f. of the price (respectively, pseudo-price) of microgrid $i$ is $\psi_{i}($.$) (respectively,$ $\left.\phi_{i}().\right)$. In Theorem 1 below, we show that the NE strategies must have a particular structure. Before stating Theorem 1, we describe some basic properties of the NE strategies.

Property $1: \phi_{2}(),. \ldots, \phi_{n}($.$) are continuous on [c, v] . \phi_{1}($. is continuous at every $x \in[c, v)$, has a jump ${ }^{5}$ of size $q_{1}-q_{2}$ at $v$ if $q_{1}>q_{2}$ and is continuous at $v$ if $q_{1}=q_{2}$.

Thus, there does not exist a pure strategy NE (one in which every microgrid selects a single price with probability (w.p.) 1).

Now, let $u_{i, \max }$ be the expected payoff that microgrid $i$ gets in the NE and $L_{i}$ be the lower endpoint of the support set ${ }^{6}$ of $\psi_{i}($.$) , i.e.:$

$$
L_{i}=\inf \left\{x: \psi_{i}(x)>0\right\} .
$$

Definition 1: Let $N_{-i}$ (respectively, $K_{-i}$ ) be the number of microgrids out of microgrids $\{1, \ldots, n\} \backslash i$ who have 1 unit of excess power (respectively, deficit power). Also, let $w_{i}=$ $P\left(N_{-i} \geq K_{-i}\right)$.

Property 2: $L_{1}=\ldots L_{n}=\tilde{p}$, where

$$
\tilde{p}=c+(v-c) \frac{1-w_{1}}{1-(1-s)^{n-1}}
$$

\footnotetext{
${ }^{4}$ The choice $v+1$ is arbitrary. Any other choice greater than $v$ also works. ${ }^{5}$ A d.f. $f(x)$ is said to have a jump (discontinuity) of size $b>0$ at $x=a$ if $f(a)-f(a-)=b$, where $f(a-)=\lim _{x \uparrow a} f(x)$ [5].

${ }^{6}$ The support set of a d.f. is the smallest closed set such that its complement
} has probability zero under the d.f. [5]. 
Also,

$$
u_{i, \max }=(\tilde{p}-c)\left[1-(1-s)^{n-1}\right], i=1, \ldots, n .
$$

Thus, the lower endpoints of the support sets of the d.f.s $\psi_{1}(),. \ldots, \psi_{n}($.$) of all the microgrids are the same and they$ get the same expected payoff in the NE.

Theorem 1: The following are necessary conditions for strategies $\phi_{1}(),. \ldots, \phi_{n}($.$) to constitute a NE:$

1) $\phi_{1}(),. \ldots, \phi_{n}($.$) satisfy Property 1$ and Property 2 .

2) There exist numbers $R_{j}, j=1, \ldots, n+1$, and a function $\{\phi(x): x \in[\tilde{p}, v)\}$ such that

$$
\begin{gathered}
\tilde{p}=R_{n+1}<R_{n} \leq R_{n-1} \leq \ldots \leq R_{1} \leq v, \\
\phi_{1}(x)=\ldots=\phi_{j}(x)=\phi(x), \tilde{p} \leq x<R_{j}, j \in\{1, \ldots, n\}, \\
\quad \text { and } \phi_{j}\left(R_{j}\right)=q_{j}, j=1, \ldots, n .
\end{gathered}
$$

Also, every point in $\left[\tilde{p}, R_{j}\right)$ is a best response for microgrid $j$ and it plays every sub-interval in $\left[\tilde{p}, R_{j}\right)$ with positive probability. Finally, $R_{1}=R_{2}=v$.

Theorem 1 says that all $n$ microgrids play prices in the range $\left[\tilde{p}, R_{n}\right)$, the d.f. $\phi_{n}($.$) of microgrid n$ stops increasing at $R_{n}$, the remaining microgrids $1, \ldots, n-1$ also play prices in the range $\left[R_{n}, R_{n-1}\right)$, the d.f. $\phi_{n-1}($.$) of microgrid n-1$ stops increasing at $R_{n-1}$, and so on. Also, microgrid 1's d.f. $\phi_{1}($.$) has a jump of height q_{1}-q_{2}$ at $v$ if $q_{1}>q_{2}$. Fig. 1 illustrates the structure.

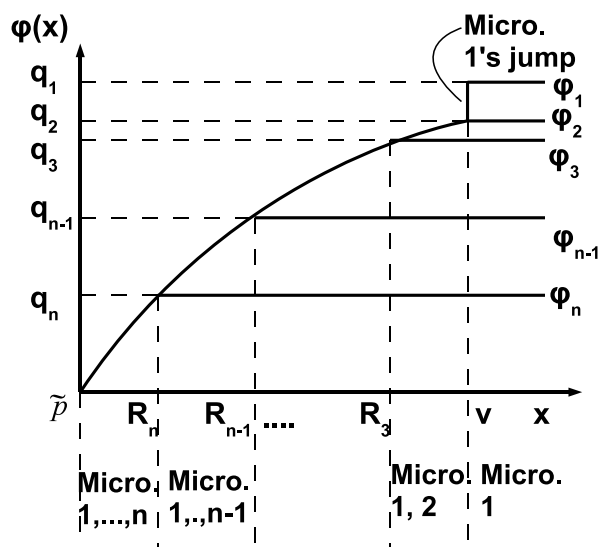

Fig. 1. The figure shows the structure of a NE described in Theorem 1. The horizontal axis shows prices in the range $x \in[\tilde{p}, v]$ and the vertical axis shows the functions $\phi($.$) and \phi_{1}(),. \ldots, \phi_{n}($.$) .$

\section{B. Explicit Computation, Uniqueness and Sufficiency}

By Theorem 1, for each $i \in\{1, \ldots, n\}$ :

$$
\phi_{i}(x)= \begin{cases}\phi(x), & \tilde{p} \leq x<R_{i} \\ q_{i}, & x \geq R_{i}\end{cases}
$$

So the candidate NE strategies $\phi_{1}(),. \ldots, \phi_{n}($.$) are completely$ determined once $\tilde{p}, R_{1}, \ldots, R_{n}$ and the function $\phi($.$) are$ specified. Also, Property 2 provides the value of $\tilde{p}$, and $R_{1}=R_{2}=v$ by Theorem 1 . First, we will show that there also exist unique $R_{3}, \ldots, R_{n}$ and $\phi($.$) satisfying (5),$ (6), and (7) and will compute them. Then, we will show that the resulting strategies given by (8) indeed constitute a $\mathrm{NE}$ (sufficiency).
Definition 2: Let $p_{-i}^{\prime}$ be the $K_{-i}$ 'th smallest pseudo-price out of the pseudo-prices, $\left\{p_{l}^{\prime}: l \in\{1, \ldots, n\}, l \neq i\right\}$, of the microgrids other than $i$ (with $p_{-i}^{\prime}=0$ if $K_{-i}=0$ ). Also, let $F_{-i}(x)$ denote the d.f. of $p_{-i}^{\prime}$.

Since $K_{-1}$ microgrids out of microgrids $2, \ldots, n$ have deficit power, if microgrid 1 has excess power and sets $p_{1}=x \in[\tilde{p}, v)$, its power is bought iff $^{7} p_{-1}^{\prime}>x$, which happens w.p. $1-F_{-1}(x)$. Note that microgrid 1's payoff is $(x-c)$ if its power is bought and 0 otherwise. So, letting $E\left\{u_{i}\left(x, \psi_{-i}\right)\right\}$ denote the expected payoff of microgrid $i$ if it sets a price $x$ and the other microgrids use the strategy profile $\psi_{-i}$, we have that for $x \in[\tilde{p}, v)$ :

$$
\begin{aligned}
E\left\{u_{1}\left(x, \psi_{-1}\right)\right\} & =(x-c)\left(1-F_{-1}(x)\right) \\
& =(\tilde{p}-c)\left[1-(1-s)^{n-1}\right]
\end{aligned}
$$

where the second equality follows from the facts that each $x \in[\tilde{p}, v)$ is a best response for microgrid 1 by Theorem 1 , and $u_{1, \max }=(\tilde{p}-c)\left[1-(1-s)^{n-1}\right]$ by (4). By (9), we get:

$$
F_{-1}(x)=g(x), x \in[\tilde{p}, v) \text {. }
$$

where, $g(x)=\frac{x-c-(\tilde{p}-c)\left[1-(1-s)^{n-1}\right]}{x-c}, x \in[\tilde{p}, v)$.

Next, we calculate $R_{i}, i=3, \ldots, n$ and $\phi($.$) using (10).$

1) Computation of $R_{i}, i=3, \ldots, n$ :

Definition 3: Consider $n-1$ events, each of which has three possible outcomes- deficit, success and failure. Each event results in deficit w.p. $s$. Let $K_{-1}$ be the total number of events that result in deficit ${ }^{8}$. For $0 \leq y \leq 1$, let $f_{i}(y)$ be the probability of $K_{-1}$ or more successes out of the $n-1$ events if $i-1$ of them have success probability $y$ and the remaining $n-i$ have success probabilities $q_{i+1}, \ldots, q_{n}$.

An expression for $f_{i}($.$) can be easily computed, using which$ we prove in the Appendix that:

Lemma 1: $f_{i}($.$) is a continuous and strictly increasing func-$ tion.

Now, to compute $R_{i}, i \in\{3, \ldots, n\}$, we note that by (8) and (5), $\phi_{j}\left(R_{i}\right)=q_{i}, j=2, \ldots, i$, and $\phi_{j}\left(R_{i}\right)=q_{j}, j=$ $i+1, \ldots, n$. Also, we define the $n-1$ events in the preceding paragraph as follows: for $j \in\{2, \ldots, n\}$, let the $j$ 'th event result in deficit if the $j$ 'th microgrid has 1 unit of deficit power, in success if $\left\{p_{j}^{\prime} \leq R_{i}\right\}$ and in failure otherwise. Then by the definition of $F_{-1}($.$) , we get:$

$$
F_{-1}\left(R_{i}\right)=f_{i}\left(q_{i}\right) .
$$

By (10) and (12):

$$
g\left(R_{i}\right)=f_{i}\left(q_{i}\right) .
$$

By (11) and (13), $R_{i}$ is unique and is given by:

$$
R_{i}=c+\frac{(\tilde{p}-c)\left[1-(1-s)^{n-1}\right]}{1-f_{i}\left(q_{i}\right)} .
$$

We now verify that the expression for $R_{i}$ in (14) is consistent with the necessity condition in (5) in Theorem 1. First,

\footnotetext{
${ }^{7}$ By Property 1 , no microgrid has a jump at any $x \in[\tilde{p}, v)$. So $P\left(p_{-1}^{\prime}=\right.$ $x)=0$.

${ }^{8}$ Note that we have defined $K_{-1}$ twice since we had previously defined it to be the number of microgrids out of microgrids $2, \ldots, n$ who have 1 unit of deficit power. However, when we use the function $f_{i}($.$) , there will be no$ conflict between the two definitions, since we will define the $n-1$ events so that the two definitions match.
} 
we show that $R_{i} \geq R_{i+1}$ for $i \in\{3, \ldots, n-1\}$. From Definition 3 and since $q_{i} \geq q_{i+1}$ by (1), it is easy to check that $f_{i}\left(q_{i}\right) \geq f_{i+1}\left(q_{i+1}\right)$. So by (14), $R_{i} \geq R_{i+1}$. Now, by Definition 3 , it follows that $f_{n}\left(q_{n}\right)>P\left(K_{-1}=0\right)=(1-s)^{n-1}$; so by (14), $R_{n}>\tilde{p}$. Also, by Definitions 1 and 3 and by (1), it follows that $w_{1} \geq f_{3}\left(q_{3}\right)$. Hence, by (3) and (14), it follows that $R_{3} \leq v$. Thus, (14) is consistent with (5).

2) Computation of $\phi($.$) : Now we compute the function$ $\{\phi():. x \in[\tilde{p}, v)\}$ by separately computing it for each interval $\left[R_{i+1}, R_{i}\right), i \in\{2, \ldots, n\}$. If $R_{i+1}=R_{i}$, then note that the interval $\left[R_{i+1}, R_{i}\right)$ is empty. Now suppose $R_{i+1}<R_{i}$. For $x \in\left[R_{i+1}, R_{i}\right)$, by (8) and (5):

$$
\begin{gathered}
\phi_{j}(x)=q_{j}, j=i+1, \ldots, n \\
\text { and } \phi_{1}(x)=\ldots=\phi_{i}(x)=\phi(x) .
\end{gathered}
$$

We define the $n-1$ events in the definition of the function $f_{i}($.$) as follows: for j \in\{2, \ldots, n\}$, let the $j$ 'th event result in deficit if the $j$ 'th microgrid has 1 unit of deficit power, in success if $\left\{p_{j}^{\prime} \leq x\right\}$ and in failure otherwise. By definition of $F_{-1}(x)$ and using $P\left\{p_{j}^{\prime} \leq x\right\}=\phi_{j}(x)$, (15) and (16):

$$
F_{-1}(x)=f_{i}(\phi(x)), R_{i+1} \leq x<R_{i} .
$$

By (10) and (17):

$$
f_{i}(\phi(x))=g(x), R_{i+1} \leq x<R_{i} .
$$

Lemma 2: For each $x$, with $R_{n+1}=\tilde{p}, R_{n}, \ldots, R_{3}$ given by (14) and $R_{2}=R_{1}=v$, (18) has a unique solution $\phi(x)$. The function $\phi($.$) is strictly increasing and continuous on$ $[\tilde{p}, v)$. For $i \in\{2, \ldots, n\}, \phi\left(R_{i}\right)=q_{i}$. Also, $\phi(\tilde{p})=0$.

Thus, there is a unique function $\phi($.$) , and by (8), unique$ $\phi_{i}(),. i=1, \ldots, n$ that satisfy the conditions in Theorem 1.

\section{3) Sufficiency:}

Theorem 2: The pseudo-price d.f.s $\phi_{i}(),. i=1, \ldots, n$ in (8), with $R_{1}=R_{2}=v, R_{i}, i=3, \ldots, n$ given by (14), and $\phi($.$) being the solution of (18), constitute the unique NE.$ The corresponding price d.f.s are $\psi_{i}(x)=\frac{1}{q_{i}} \phi_{i}(x), x \in[c, v]$, $i=1, \ldots, n$.

\section{Discussion}

The structure of the unique NE identified in Theorems 1 and 2 provides several interesting insights:

1) First, by Property $1, \psi_{1}($.$) has a jump at v$ iff $q_{1}>q_{2}$ and is continuous everywhere else, whereas $\psi_{2}(),. \ldots, \psi_{n}($.$) are$ always continuous on $[c, v]$. Thus, each microgrid randomizes over a range of prices. This random selection of prices can be interpreted as follows: each microgrid $i$ that has excess power sets a base price $v$ and randomly holds "sales" to attract the microgrids that have deficit power by lowering the price to some value $p_{i}<v^{9}$.

2) Second, from (1), (5) and the fact that the support set of $\psi_{i}($.$) is \left[\tilde{p}, R_{i}\right]$, it follows that only the microgrids with a high excess power availability probability $(q)$ play high prices (see Fig. 1). Intuitively this is because all the microgrids play low prices (near $\tilde{p}$ ), so if a microgrid sets a high price, it is undercut by all the other microgrids. But a microgrid with a high $q$ runs a lower risk of being undercut than one with a low $q$ because of the lower excess power availability probabilities of the set

\footnotetext{
${ }^{9}$ This interpretation has been suggested in [7] for random selection of prices in a different context.
}

of microgrids other than itself.

3) Third, note that there does not exist a pure strategy NE, and the unique NE is of mixed-strategy type. We contrast this with the Bertrand price competition game [8], in which (i) there are $n$ sellers, each of whom owns 1 unit of a good w.p. 1 , (ii) there are $k \in\{1, \ldots, n-1\}$ buyers (distinct from the sellers), each of whom needs 1 unit of the good w.p. 1, (iii) each seller $i$ sets a price $p_{i} \in[c, v]$, where $c<v$ and (iv) the utility $u_{i}\left(p_{1}, \ldots, p_{n}\right)$ of seller $i$ if seller $j$ sets a price $p_{j}, j=1, \ldots, n$, is $p_{i}-c$ if seller $i$ 's good is bought and 0 otherwise. Note that the game in our paper differs from the Bertrand game in that there is uncertainty in the availability of the goods with the sellers, and the sets of buyers and sellers are drawn from a common pool of traders. In the Bertrand game, the pure strategy profile under which each seller deterministically selects $c$ as his price is the unique NE [8]. This strategy profile is not a NE in our context as it provides 0 utility for each microgrid, whereas by quoting any price above $c$ (and below $v$ ), each microgrid with excess power can attain a positive expected utility since it will sell its power at least in the event that it is the only microgrid with excess power and at least one microgrid has deficit power, which happens with positive probability. Thus, uncertainty in the availability of goods with the sellers and the drawing of the sets of buyers and sellers from a common pool of traders fundamentally alters the structure of the NE.

4) Finally, we compare the NE found in this section with that in the game studied in [13], which is like the Bertrand game described in 3) above, with the difference that each seller $i \in\{1, \ldots, n\}$ owns 1 unit of the good w.p. $q_{i} \in(0,1)$ (instead of w.p. 1) and 0 units w.p. $1-q_{i}$. Note that in the game studied in this section as well as in the game in [13], there is uncertainty in the availability of the goods with the sellers. However, the difference between the two games is that in the former, the sets of buyers and sellers are drawn from the same pool of traders, whereas in the latter, the sets of buyers and sellers are distinct. Recall that in the former game, (i) for the case $n=2$, as noted at the end of Section II, the strategy profile in which both microgrids $i=1,2$ set the price $p_{i}=v$ w.p. 1 is the unique $\mathrm{NE}$ and (ii) for the case $n \geq 3$, the structure of the unique NE is as in Theorems 1 and 2 . However, in the latter game, the structure of the NE for $n \geq 3$ as well as for $n=2$ is similar to the structure in Theorems 1 and 2 [13].

\section{Asymmetric Deficit Power Probabilities}

In Section III, we assumed that $s_{1}=\ldots=s_{n}=s$ for some $s \in(0,1)$. In this section, we relax that assumption and allow $s_{1}, \ldots, s_{n}$ to be unequal. This makes the analysis significantly harder. So for tractability, we consider only the case $n=3$ and find a NE.

In this section, we do not assume that (1) holds, but instead, consider a generalization of that condition. Consider the quantity $\frac{1}{q_{i}}\left(1-\frac{s_{i}}{2}\right), i \in\{1,2,3\}$. Without loss of generality, assume that $i=3$ maximizes it, i.e.:

$$
\frac{1}{q_{3}}\left(1-\frac{s_{3}}{2}\right)=\max _{i=1,2,3} \frac{1}{q_{i}}\left(1-\frac{s_{i}}{2}\right) .
$$

Also, suppose:

$$
q_{3}\left(s_{2}-s_{1}\right)+s_{3}\left(q_{2}-q_{1}\right) \leq\left(1-s_{3}\right)\left(q_{1} s_{2}-q_{2} s_{1}\right) .
$$


Note that the conditions in (19) and (20) together generalize condition (1), and they reduce to (1) when $s_{1}=s_{2}=s_{3}=s$. In the sequel, we will present a strategy profile that is a NE when (19) and (20) hold. When (20) does not hold, then the strategy profile obtained by swapping the roles of microgrids 1 and 2 everywhere in the above strategy profile is a NE.

\section{A. The NE}

Let $p_{i}$ be the price selected by microgrid $i$ and let the corresponding pseudo-price $p_{i}^{\prime}$ be as defined in Section III. Also, as before, let $\psi_{i}($.$) and \phi_{i}($.$) be the d.f. of p_{i}$ and $p_{i}^{\prime}$ respectively. Let $L_{i}$ (respectively, $R_{i}$ ) be the left (respectively, right) endpoint of the support set of $\psi_{i}($.$) .$

We will now describe the NE strategies. Let

$$
\tilde{p}=c+\left\{1-\frac{\left(s_{3} q_{2}+s_{2} q_{3}\right)}{\left(s_{3}+s_{2}-s_{3} s_{2}\right)}\right\}(v-c)
$$

It is easy to check that $c<\tilde{p}<v$. We will later see that in the NE, $L_{1}=L_{2}=L_{3}=\tilde{p}$. Also, $\tilde{p}<R_{3} \leq R_{2}=R_{1}=v$, where:

$$
R_{3}=c+\frac{(\tilde{p}-c)}{1-\frac{q_{3}}{1-\frac{s_{3}}{2}}}
$$

Let:

$$
F(x)=\frac{x-\tilde{p}}{x-c}
$$

$$
\begin{aligned}
& \psi_{1}(x)= \\
& \begin{cases}\frac{1}{q_{1}}\left(1-\frac{s_{1}}{2}\right) F(x), & \tilde{p} \leq x<R_{3} \\
\frac{1}{s_{3} q_{1}}\left\{\left(s_{1}+s_{3}-s_{1} s_{3}\right) F(x)-s_{1} q_{3}\right\}, & R_{3} \leq x<v \\
1, & x \geq v\end{cases}
\end{aligned}
$$

$$
\begin{aligned}
& \psi_{2}(x)= \\
& \begin{cases}\frac{1}{q_{2}}\left(1-\frac{s_{2}}{2}\right) F(x), & \tilde{p} \leq x<R_{3} \\
\frac{1}{s_{3} q_{2}}\left\{\left(s_{2}+s_{3}-s_{2} s_{3}\right) F(x)-s_{2} q_{3}\right\}, & R_{3} \leq x<v \\
1, & x \geq v\end{cases}
\end{aligned}
$$

$$
\psi_{3}(x)= \begin{cases}\frac{1}{q_{3}}\left(1-\frac{s_{3}}{2}\right) F(x), & \tilde{p} \leq x<R_{3} \\ 1 & x \geq R_{3}\end{cases}
$$

Theorem 3: The strategies $\psi_{1}(),. \psi_{2}($.$) and \psi_{3}($.$) in (24),$ (25) and (26) constitute a NE.

\section{B. Discussion}

It can be checked that when $s_{1}=s_{2}=s_{3}=s$, the NE strategies in (24), (25) and (26) reduce to those computed in Section III.

We now compare the structure of the NE given by (24), (25) and (26) with that in Theorem 1. First, note that by (24), (25) and (26), for $x$ in the range $\left[\tilde{p}, R_{3}\right)$, each of $\psi_{1}(x), \psi_{2}(x)$ and $\psi_{3}(x)$ equals $F(x)$ times a constant factor (i.e., a factor that does not depend on $x$ ), and hence they differ only by a constant multiplicative factor. This is similar to the NE strategies in Theorem 1 (with $n=3$ ), for which, by (6) and the fact that $\phi_{i}(x)=q_{i} \psi_{i}(x)$, each of $\psi_{1}(x), \psi_{2}(x)$ and $\psi_{3}(x)$ equals $\phi(x)$ times a constant factor on $x \in\left[\tilde{p}, R_{3}\right)$. However, a difference is that for the NE strategies in Theorem 1 , for $x$ in the range
$\left[R_{3}, v\right), \psi_{1}($.$) and \psi_{2}($.$) differ only by a constant multiplicative$ factor, whereas this is not the case in general for the NE in (24), (25) and (26). Thus, a structure similar to that in (6) does not hold in general for the NE in (24), (25) and (26).

Property 1 generalizes to the NE in (24), (25) and (26) as follows. $\phi_{2}($.$) and \phi_{3}($.$) are continuous on [c, v] . \phi_{1}($. is continuous at every $[c, v)$; it is continuous at $v$ if (20) holds with equality and has a jump at $v$ otherwise, whose size can be obtained from (24). Property 2 generalizes to give the following. $L_{1}=\ldots=L_{n}=\tilde{p}$, where $\tilde{p}$ is given by (21). Also, the expected payoffs of the microgrids in the NE are given by:

$$
\begin{gathered}
u_{1, \max }=(\tilde{p}-c)\left(s_{2}+s_{3}-s_{2} s_{3}\right), \\
u_{2, \max }=(\tilde{p}-c)\left(s_{1}+s_{3}-s_{1} s_{3}\right) \\
\text { and } u_{3, \max }=(\tilde{p}-c)\left(s_{1}+s_{2}-s_{1} s_{2}\right) .
\end{gathered}
$$

Note that when $s_{1}=s_{2}=s_{3}$, by Property 2 , the expected payoffs of the three microgrids in the NE are equal. Also, recall that in point 4 in the discussion in Section III-C, we noted that the structure of the unique NE in the game studied in [13] is similar to that in Theorems 1 and 2; it also turns out that the expected payoffs of all the sellers in that $\mathrm{NE}$ are equal [13]. However, for the NE in (24), (25) and (26), the expected payoffs of the three microgrids are not equal in general, as can be seen from (27), (28) and (29). This is an interesting idiosyncrasy brought about by the inequity among $s_{1}, s_{2}$ and $s_{3}$.

\section{RANDOM $v$}

In Sections III and IV, we assumed that $v$, the price at which the macrogrid sells unit power, is constant and known to the microgrids. However, this need not always be the case in practice. So in this section, we analyze the scenario where $v$ is a random variable whose value is unknown to the microgrids. Suppose there are $n$ microgrids, where $n \geq 3$. For simplicity, as in Section III, we assume that (i) $s_{1}=\ldots=s_{n}=s$ for some $s \in(0,1)$ and (ii) $c$ is a constant that is known to the microgrids. Also, as in Section III, assume without loss of generality that (1) holds.

Suppose $v$ takes values in the interval $[\underline{v}, \bar{v}]$ w.p. 1 , where $c<\underline{v}<\bar{v}$. Note that the price $v$ at which the macrogrid sells unit power is always upper bounded in practice by some finite constant $\bar{v}$. Also, as we mentioned in Section III, the macrogrid always sells power at a higher price than the price $c$ at which it buys power, due to redistribution costs and since it makes a profit; so $\underline{v}>c$.

Let $G($.$) be the d.f. of v$. We assume that $G($.$) is known to$ all the microgrids. Also, let $h(x)=(x-c)(1-G(x))$. For tractability, we make the following technical assumption on $G($.$) :$

Assumption 1: $G($.$) is continuous. Also, the function h($. has a unique maximizer, say $v_{T}$, and $h($.$) is strictly increasing$ on the interval $\left[c, v_{T}\right]$.

Note that a large class of distribution functions $G($.$) , including$ the uniform distribution on $[\underline{v}, \bar{v}]$, satisfy the above assumption.

Similar to the constant $v$ case, we define the pseudo-price of microgrid $i, p_{i}^{\prime}$, as the price $p_{i}$ it selects if it has 1 unit of excess power and $p_{i}^{\prime}=\bar{v}+1$ otherwise. Also, let $\psi_{i}($.$) (respectively,$ 
$\left.\phi_{i}().\right)$ be the d.f. of $p_{i}$ (respectively, $\left.p_{i}^{\prime}\right)$. As before, $\phi_{i}(x)=$ $q_{i} \psi_{i}(x)$.

As before, let $K_{-i}$ be the number of microgrids out of $\{1, \ldots, n\} \backslash i$ who have 1 unit of deficit power and let $p_{-i}^{\prime}$ be as in Definition 2. Now, if a microgrid $i$ sets a price $p_{i}$, then its power is sold iff (i) $v \geq p_{i}$ (if $v<p_{i}$, then a microgrid with deficit power would prefer to buy power from the macrogrid instead of from microgrid $i$ ) and (ii) microgrid $i$ is one of the microgrids with the $K_{-i}$ lowest pseudo-prices (and among the randomly selected ones in case of ties in pseudo-prices); let the probability of the event in (ii) be $B\left(p_{i}\right)$. So microgrid $i$ 's expected revenue is:

$$
\begin{aligned}
E\left\{u_{i}\left(p_{i}, \psi_{-i}\right)\right\} & =\left(p_{i}-c\right) P\left(p_{i} \leq v\right) B\left(p_{i}\right) \\
& =\left(p_{i}-c\right)\left(1-G\left(p_{i}\right)\right) B\left(p_{i}\right) \\
& =h\left(p_{i}\right) B\left(p_{i}\right)
\end{aligned}
$$

Now, by Assumption 1, $h($.$) has a unique maximizer at v_{T}$. Also, by definition, the function $B\left(p_{i}\right)$ is a nonincreasing function of $p_{i}$. So by (30), $E\left\{u_{i}\left(p_{i}, \psi_{-i}\right)\right\}<E\left\{u_{i}\left(v_{T}, \psi_{-i}\right)\right\}$ for all $p_{i}>v_{T}$ and hence no microgrid sets a price greater than $v_{T}$. In fact, as the analysis below shows, $v_{T}$ plays the role that $v$ plays in the constant $v$ case in Section III.

Now, since $\underline{v} \leq v \leq \bar{v}, G\left(p_{i}\right)=1$ for $p_{i} \geq \bar{v}$. So

$$
h\left(p_{i}\right)=0, p_{i} \geq \bar{v} .
$$

Also, $G\left(p_{i}\right)=0$ for $p_{i} \leq \underline{v}$. So $h\left(p_{i}\right)=p_{i}-c$ for $c \leq p_{i} \leq \underline{v}$, which is a strictly increasing function of $p_{i}$ and $h(\underline{v})=\underline{v}-c>$ 0 . This, combined with (31), gives:

$$
\underline{v} \leq v_{T}<\bar{v} .
$$

Next, we explicitly compute a NE and show that it is unique. The results are similar to those in the constant $v$ case in Section III, and hence we only state the differences.

First, we state some necessary conditions that the NE strategies must satisfy. Property 1 in Section III-A holds in the present context with $v_{T}$ in place of $v$. Let $w_{i}$ be as in Definition 1.

Lemma 3: The equation

$$
h(x)=\frac{h\left(v_{T}\right)\left(1-w_{1}\right)}{\left[1-(1-s)^{n-1}\right]}
$$

has a unique solution $x \in\left(c, v_{T}\right)$. Let $\tilde{p}$ be this solution. Let

$$
u_{i, \max }=h(\tilde{p})\left[1-(1-s)^{n-1}\right] .
$$

Property 2 in Section III-A holds except that now, $\tilde{p}$ and $u_{i, \max }$ are as defined above. Also, Theorem 1 holds with Properties 1 and 2 and $\tilde{p}$ as described above, and $v_{T}$ in place of $v$.

Equation (8) holds in the present context, and to completely determine the NE strategies $\phi_{1}(),. \ldots, \phi_{n}($.$) , it remains to$ specify $R_{3}, \ldots, R_{n}$ and the function $\phi($.$) . Let F_{-i}($.$) be as$ in Section III. Similar to the derivation of (9) and using (30) and (34), we get that for $x \in\left[\tilde{p}, v_{T}\right)$ :

$E\left\{u_{1}\left(x, \psi_{-1}\right)\right\}=h(x)\left(1-F_{-1}(x)\right)=h(\tilde{p})\left[1-(1-s)^{n-1}\right]$

By (35), we get

$$
F_{-1}(x)=g(x), x \in[\tilde{p}, v),
$$

where,

$$
g(x)=\frac{h(x)-h(\tilde{p})\left[1-(1-s)^{n-1}\right]}{h(x)} .
$$

Next, we compute $R_{i}, i=3, \ldots, n$ and the function $\phi($.$) using$ (36) and (37). We define the function $f_{i}($.$) as in Section III and$ the $n-1$ events in the definition of $f_{i}($.$) as in the derivation$ of (14). Similar to the derivation of (13), we get:

$$
g\left(R_{i}\right)=f_{i}\left(q_{i}\right),
$$

which, using (37), becomes:

$$
h\left(R_{i}\right)=\frac{h(\tilde{p})\left[1-(1-s)^{n-1}\right]}{1-f_{i}\left(q_{i}\right)} .
$$

Lemma 4: Equation (38) has a unique solution $R_{i} \in\left[\tilde{p}, v_{T}\right]$. Also, similar to the derivation of (18), for each $i \in$ $\{2, \ldots, n\}$ such that $R_{i+1}<R_{i}$ :

$$
f_{i}(\phi(x))=g(x), R_{i+1} \leq x<R_{i} .
$$

Finally, Lemma 2 holds in the present context with (39) in place of (18) and $v_{T}$ in place of $v$ in the statement of the lemma and Theorem 2 holds with $R_{i}, i \in\{3, \ldots, n\}$ being the solutions of (38) instead of the values in (14), (39) in place of (18) and $v_{T}$ in place of $v$ in the statement of the theorem.

Thus, a unique NE exists and the above discussion identifies a procedure for computing it. Also, the NE has a structure similar to that in Section III; in particular, the discussion in Section III-C applies to it.

\section{NUMERICAL STUdiES}

In this section, using numerical experiments, we compare the trade of power among interconnected microgrids proposed in this paper with a scheme in which microgrids only trade power with the macrogrid, and also further study the NE studied in Sections III and IV. Throughout, we use the parameter values $c=0$ and $v=1$.

First, we consider $q_{1}, \ldots, q_{n}$ that are uniformly spaced in $\left[q_{L}, q_{H}\right]$ for some parameters $q_{L}$ and $q_{H}$, and $s_{1}=\ldots=s_{n}=$ $s$. Let $q=\frac{q_{L}+q_{H}}{2}$ be the mean probability of having excess power of the microgrids. We consider two schemes: (i) the scheme considered in this paper; and (ii) a centralized scheme in which a microgrid who has deficit power (respectively, excess power) in a slot buys power from (respectively, sells power to) the macrogrid alone. Recall that in scheme (i), a microgrid with excess power sells power to a microgrid with deficit power for a price in $[c, v]$, whereas the macrogrid buys power at price $c$ and sells power at price $v$; thus, the microgrids trade power among themselves whenever possible, and with the macrogrid only in the event of necessity arising from a mismatch between the amounts of deficit and excess power with the microgrids. Let $T_{D}$ and $T_{C}$ be the total expected power traded (bought or sold) by all the microgrids with the macrogrid in one slot in schemes (i) and (ii) respectively. Note that since the microgrids are close to each other, power that is traded by the microgrids with the macrogrid is typically transmitted over longer distances than the power that is traded among the microgrids, resulting in larger transmission losses. Fig 2 plots $T_{D}, T_{C}$ and the ratio $\eta=\frac{T_{D}}{T_{C}}$ versus $q$, and shows that scheme (i) results in considerable savings in the total 
expected power exchanged with the macrogrid over scheme (ii) (the savings are between $43.4 \%$ and $68.4 \%$ in the current example). Also, note that $T_{D}$ achieves its lowest value around $q=0.25$, which is close to the value of $s(0.3)$. This is consistent with the intuition that when the mean excess power and deficit power probabilities of the microgrids are close to each other, the average total excess power available with the microgrids with excess power roughly matches the average total deficit power required by the microgrids with deficit power, and hence only a small amount of power needs to be exchanged with the macrogrid. Thus, trade of power among interconnected microgrids can result in substantial savings in the amount of power that needs to be transmitted over large distances, especially when the average excess power and deficit power probabilities of the microgrids are close to each other.

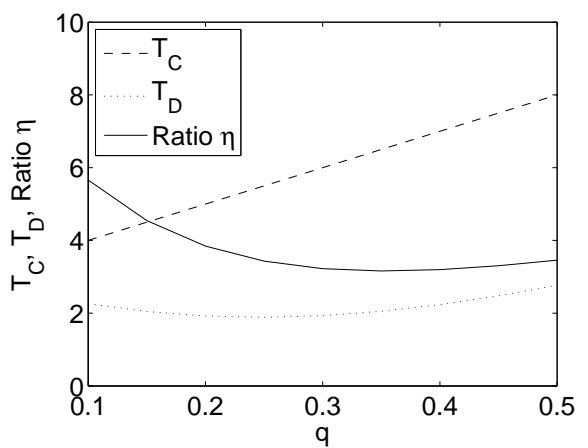

Fig. 2. The figure plots $T_{C}, T_{D}$ and $\eta=\frac{T_{D}}{T_{C}}$ versus $q$. The parameter values used are $n=10, q H-q L=0.2$ and $s=0.3 . \eta$ is scaled by a factor of 10 in order to show it on the same figure as the other plots.

Now, we consider the model analyzed in Section III with the parameter values $n=4, q_{1}=0.6, q_{2}=0.5, q_{3}=0.4, q_{4}=$ 0.3 and $s=0.3$. For these parameters, the top plot in Fig. 3 shows the price selection d.f.s of the microgrids in the NE in Theorems 1 and 2. The structure of the functions in the plot is as in Theorem 1 with $\tilde{p}=0.45, R_{1}=R_{2}=1, R_{3}=0.93$ and $R_{4}=0.82$. In particular, microgrid 1's price selection d.f. has a jump at $v$. Next, we consider the model analyzed in Section IV with the parameter values $n=3, q_{1}=0.6$, $q_{2}=0.5, q_{3}=0.4, s_{1}=0.3, s_{2}=0.2$ and $s_{3}=0.1$. The bottom plot in Fig. 3 shows the price selection d.f.s of the microgrids in the NE for these parameter values. Their structure is as found in Section IV (see (24), (25) and (26)) with $\tilde{p}=0.54, R_{1}=R_{2}=1$ and $R_{3}=0.92$. In particular, microgrid 1's price selection d.f. has a jump at $v$. The two plots in Fig. 3 shows that the price selection d.f.s in the NE with symmetric and asymmetric deficit power probabilities are qualitatively similar.

Finally, we again consider the model in Section III with $q_{1}, \ldots, q_{n}$ that are uniformly spaced in $\left[q_{L}, q_{H}\right]$ for some parameters $q_{L}$ and $q_{H}$. Let $q=\frac{q_{L}+q_{H}}{2}$ be the mean probability of having excess power of the microgrids. Fig. 4 plots the mean price of excess power quoted by microgrid 1 in the NE found in Section III versus $q$. The figure shows that the mean price is decreasing in $q$; this is because, since $s$ is constant, as $q$ increases, the expected supply of power in the market increases relative to the expected demand for power and the price competition among the microgrids with excess power
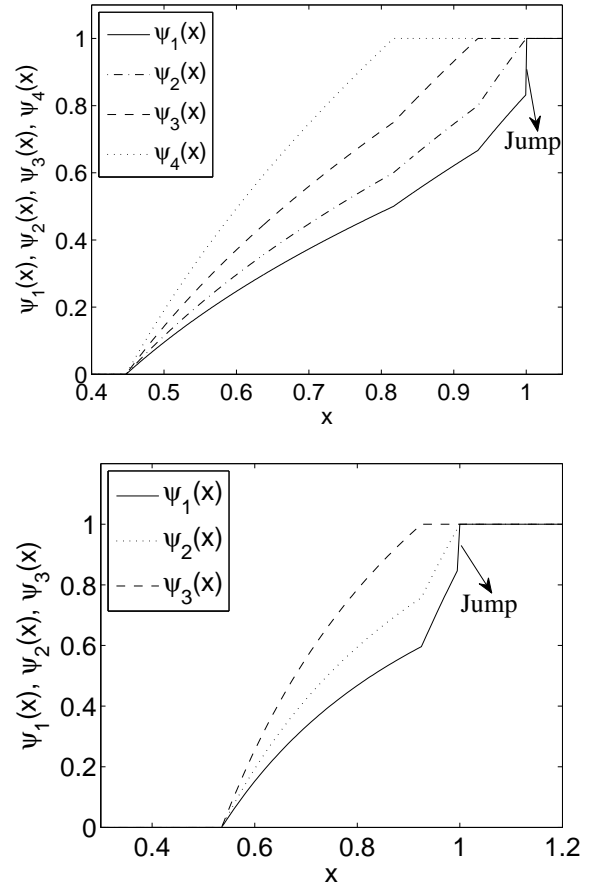

Fig. 3. The top plot shows the functions $\psi_{1}(),. \psi_{2}(),. \psi_{3}($.$) and \psi_{4}($.$) for$ the model in Section III with the parameter values in the text. The bottom plot shows the functions $\psi_{1}(),. \psi_{2}($.$) and \psi_{3}($.$) for the model in Section IV$ with the parameter values in the text.

becomes more intense, driving down the prices.

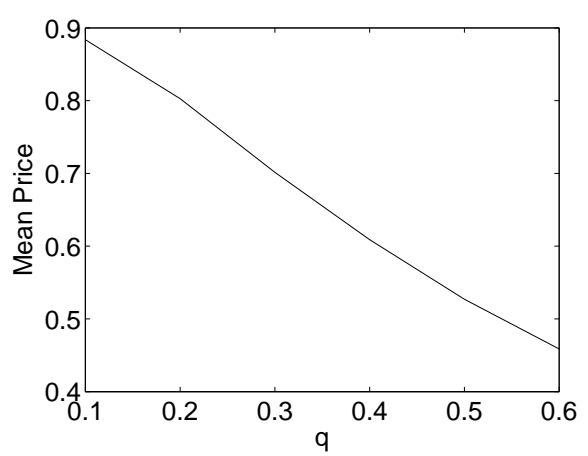

Fig. 4. The figure plots the mean price of excess power quoted by microgrid 1 versus $q$ for the parameter values $n=8, q H-q L=0.2$ and $s=0.25$.

\section{CONCLUSIONS}

We analyzed price competition among interconnected microgrids and found NE in the corresponding game. The analysis provides several insights- for example, there is randomization in the selection of prices by the microgrids who have excess power and, when the probabilities of having deficit power are symmetric, only microgrids with a high excess power availability probability set high prices. Numerical experiments showed that trade of power among interconnected microgrids results in significant savings in the total expected power transmitted over long distances and hence the transmission losses. We noted that explicit computation and an investigation of the uniqueness of the NE is complicated when the deficit 
power probabilities of the microgrids are asymmetric; in this paper, we have computed a NE for the case $n=3$. A direction for future work is to compute the NE and to investigate its uniqueness for arbitrary $n$.

\section{REFERENCES}

[1] A. Mas-Colell, M. Whinston, J. Green, "Microeconomic Theory", Oxford University Press, 1995.

[2] R. Myerson, "Game Theory: Analysis of Conflict", Harvard University Press, 1997.

[3] R.H. Lasseter, "Microgrids and Distributed Generation", Journal of Energy Engineering, Vol. 133, pp. 144-149, Sept. 2007.

[4] S.P. Chowdhury, P. Crossley, S. Chowdhury, "Microgrids and Active Distribution Networks", Institution of Engineering and Technology, 2009.

[5] B.S. Everitt, The Cambridge Dictionary of Statistics, 3rd ed., Cambridge University Press, 2006.

[6] W. Rudin, "Principles of Mathematical Analysis", Mc-Graw Hill, Third Edition, 1976.

[7] H.R. Varian, "A Model of Sales", In American Economic Review, Vol 70, pp. 651-659, 1980.

[8] J.E. Harrington, "A Re-Evaluation of Perfect Competition as the Solution to the Bertrand Price Game", In Math. Soc. Sci., Vol. 17, pp. 315-328, 1989.

[9] J. E. Walsh, "Existence of Every Possible Distribution for any Sample Order Statistic", In Statistical Papers, Vol. 10, No. 3, Springer Berlin, Sept. 1969.

[10] M. Janssen, E. Rasmusen "Bertrand Competition Under Uncertainty", In J. Ind. Econ., 50(1): pp. 11-21, March 2002.

[11] S. Kimmel "Bertrand Competition Without Completely Certain Production", Economic Analysis Group Discussion Paper, Antitrust Division, U.S. Department of Justice, 2002.

[12] G.S. Kasbekar, S. Sarkar, "Spectrum Pricing Games with Bandwidth Uncertainty and Spatial Reuse in Cognitive Radio Networks", in Proc. of MobiHoc, September 20-24, Chicago, IL, USA, 2010.

[13] G.S. Kasbekar, S. Sarkar, "Spectrum Pricing Games with Arbitrary Bandwidth Availability Probabilities", In Proc. of ISIT, St. Petersburg, Russia, July 31-August 5, 2011.

[14] J.S. John, "Balance Energy Quietly Building a Web of Microgrids", http://gigaom.com/cleantech/balance-energy-quietly-buildinga-web-of-microgrids

[15] H. Jiayi, J. Chuanwen, X. Rong, "A review on distributed energy resources and MicroGrid", Renewable and Sustainable Energy reviews, 12(9): pp. 2472-2483, Dec. 2008.

[16] S.M. Ali, "Electricity Trading among Microgrids", M.S. Thesis, Department of Mechanical Engineering, University of Strathclyde, 2009.

[17] Z. Alibhai, W. A. Gruver, D. B. Kotak, D. Sabaz, "Distributed Coordination of Micro-grids using Bilateral Contracts", In Proc. of IEEE International Conference on Systems, Man and Cybernetics, Hague, Netherlands, Oct. 2004.

[18] I.-K. Cho, S.P. Meyn, "Efficiency and Marginal Cost Pricing in Dynamic Competitive Markets with Friction", Theoretical Economics, 5(2): pp. 215-239, Dec. 2010

[19] S. Meyn, M. Negrete-Pincetic, G. Wang, A. Kowli, E. Shafieepoorfard, "The Value of Volatile Resources in Electricity Markets", In Proc. of the 49th Conference on Decisions and Control (CDC), Atlanta, GA, Dec. 2010.

[20] G. Wang, A. Kowli, M. Negrete-Pincetic, E. Shafieepoorfard, S. Meyn, "A Control Theorist's Perspective on Dynamic Competitive Equilibria in Electricity Markets", In Proceedings of the 18th World Congress of the International Federation of Automatic Control (IFAC), Milano, Italy, 2011.

[21] R. E. Bohn, M. C. Caramanis, F. C. Schweppe, "Optional Pricing in Electrical Networks over Space and Time", The RAND Journal of Economics, 15(3): pp. 360-376, Autumn 1984

[22] R. Bjorgan, H. Song, C.-C Liu, R. Dahlgren, "Pricing Flexible Electricity Contracts", IEEE Transactions on Power Systems, 15(2): pp. 477-482, May 2000.

[23] M.C. Caramanis, R.E. Bohn, F.C. Schweppe, "Optimal Spot Pricing: Practice And Theory", IEEE Transactions on Power Apparatus and Systems, 101(9): pp. 3234-3245, Sept. 1982.

[24] R. J. Kaye, H. R. Outhred, C. H. Bannister, "Forward Contracts for the Operation of an Electricity Industry under Spot Pricing", IEEE Transactions on Power Systems, 5(1): pp. 46-52, Feb. 1990.

[25] T. W. Gedra, P. P. Varaiya, "Markets and Pricing for Interruptible Electric Power", IEEE Transactions on Power Systems, 8(1): pp. 122-128, Feb. 1993.

[26] T. W. Gedra, "Optional Forward Contracts for Electric Power Markets", IEEE Transactions on Power Systems, 9(4): pp. 1766-1773, Nov. 1994.

\section{APPENDIX}

\section{A. Proofs of results in Section III-A}

We first prove a lemma (Lemma 5) that we use throughout. Next we prove Property 2 and then Property 1 and Theorem 1.

Lemma 5: For $i=1, \ldots, n, \psi_{i}($.$) is continuous, except$ possibly at $v$. Also, at most one microgrid has a jump at $v$.

Proof: Suppose $\psi_{i}($.$) has a jump at a point x_{0}, c<x_{0}<$ $v$. Then for some $\epsilon>0$, no microgrid $j \neq i$ chooses a price in $\left[x_{0}, x_{0}+\epsilon\right]$ because it can get a strictly higher payoff by choosing a price just below $x_{0}$ instead. This in turn implies that microgrid $i$ gets a strictly higher payoff at the price $x_{0}+\epsilon$ than at $x_{0}$. So $x_{0}$ is not a best response for microgrid $i$, which contradicts the assumption that $\psi_{i}($.$) has a jump at x_{0}$. Thus, $\psi_{i}($.$) is continuous at all x<v$.

Now, suppose microgrid $i$ has a jump at $v$. Then a microgrid $j \neq i$ gets a higher payoff at a price just below $v$ than at $v$. So $v$ is not a best response for microgrid $j$ and it plays it with 0 probability. Thus, at most one microgrid has a jump at $v$.

1) Proof of Property 2: We prove Property 2 in Lemmas 7 and 9. We first prove Lemma 6 , which will be used to prove Lemma 7. Let $u_{i, \max }$ and $L_{i}$ be as defined in Section III-A.

Lemma 6: For $i=1, \ldots, n, L_{i}$ is a best response for microgrid $i$.

Proof: By (2), either microgrid $i$ has a jump at $L_{i}$ or plays prices arbitrarily close to $L_{i}$ and above it with positive probability.

Case (i): If microgrid $i$ has a jump at $L_{i}$, then $L_{i}$ is a best response for $i$ because in a NE, no microgrid plays a price other than a best response with positive probability.

Case (ii): If microgrid $i$ does not have a jump at $L_{i}$, then by $(2), \psi_{i}\left(L_{i}\right)=0$. Since every microgrid selects a price in $[c, v], \psi_{i}(v)=1$. So $L_{i}<v$. So by Lemma 5 , no microgrid among $\{1, \ldots, n\} \backslash i$ has a jump at $L_{i}$. Hence, microgrid $i$ 's payoff at a price above $L_{i}$ and close enough to it is arbitrarily close to its payoff at $L_{i}$. But since microgrid $i$ does not have a jump at $L_{i}$, by (2), it plays prices just above $L_{i}$ with positive probability and they are best responses for it. So $L_{i}$ is also a best response for microgrid $i$.

Lemma 7: For some $c<\tilde{p}<v, L_{1}=\ldots L_{n}=\tilde{p}$. Also, $u_{i, \max }=(\tilde{p}-c)\left[1-(1-s)^{n-1}\right], i=1, \ldots, n$.

That is, the lower endpoint of the support set of the price distribution of every microgrid is the same.

Proof: Let $K_{-i}$ be as in Definition 1. Note that the expected payoff that a microgrid $i$ gets at a given price $p_{i}$ depends on the pseudo-price distribution functions of the microgrids other than $i$ and the distribution of $K_{-i}$. Also, since $s_{1}=\ldots=s_{n}=s$, the distribution of the random variable $K_{-i}$ for $i=1, \ldots, n$ is the same.

Now, suppose $L_{i}<L_{j}$ for some $i, j$. By Lemma $6, L_{j}$ is a best response for microgrid $j$. Now, the expected payoff that microgrid $j$ gets for $p_{j}=L_{j}$ is strictly less than the expected payoff that microgrid $i$ would get if it set $p_{i}$ to be just below $L_{j}$. This is because, if microgrids $i$ or $j$ set a price of approximately $L_{j}$, then they see the same pseudoprice distribution functions of the microgrids other than $i$ and $j$. But microgrid $j$ may be undercut by microgrid $i$, since $L_{i}<$ $L_{j}$, whereas microgrid $i$ may not be undercut by microgrid $j$. Also, microgrid $j$ 's expected payoff is strictly lowered due to this undercutting by microgrid $i$ because microgrid $j$ 's excess power is not sold in the event that microgrid $i$ has excess 
power and sets a price below $L_{j}$, and exactly one microgrid has deficit power, which happens with positive probability. Hence, $u_{i, \max }>u_{j, \max }$.

Now, by Lemma $6, L_{i}$ is a best response for microgrid $i$. If microgrid $j$ were to play price $L_{i}$, then it would get a payoff of $u_{i, \max }$. This is because, when microgrid $i$ plays price $L_{i}$, it gets payoff $u_{i, \max }$. Since $L_{j}>L_{i}$, microgrid $i$ is, w.p. 1 , not undercut by microgrid $j$. If microgrid $j$ were to set the price $L_{i}$, then w.p. 1 , it would not be undercut by microgrid $i$. Also, the pseudo-price distributions of the microgrids other than $i$ and $j$ are exactly the same from the viewpoints of microgrids $i$ and $j$. Thus, microgrid $j$ can strictly increase its payoff from $u_{j, \max }$ to $u_{i, \max }$ by playing price $L_{i}$, which contradicts the fact that $L_{j}$ is a best response for it.

Thus, $L_{i}<L_{j}$ is not possible. By symmetry, $L_{i}>L_{j}$ is not possible. So $L_{i}=L_{j}$. Let $L_{1}=\ldots=L_{n}=\tilde{p}$.

By Lemma 6, a price of $\tilde{p}$ is a best response for every microgrid $i$. Since no microgrid sets a price lower than $\tilde{p}$, a price of $\tilde{p}$ fetches a payoff of $\tilde{p}-c$ for microgrid $i$ if $K_{-i} \geq 1$ and a payoff of 0 if $K_{-i}=0$. So $u_{i, \max }=(\tilde{p}-c) P\left(K_{-i} \geq\right.$ $1)=(\tilde{p}-c)\left[1-(1-s)^{n-1}\right], i=1, \ldots, n$.

Let $w_{i}$ be as in Definition 1 . Using (1), it can be easily shown that:

$$
w_{1} \leq w_{2} \leq \ldots \leq w_{n} .
$$

We now prove Lemma 8 , which will be used to prove Lemma 9.

Lemma 8: For every $\epsilon>0$, there exist microgrids $m$ and $j, m \neq j$, such that $\psi_{m}(v-\epsilon)<1$ and $\psi_{j}(v-\epsilon)<1$.

That is, at least two microgrids play prices just below $v$ with positive probability.

Proof: Suppose not. Fix $i$ and let:

$$
y=\inf \left\{x: \psi_{l}(x)=1 \forall l \neq i\right\} .
$$

By definition of $y, \psi_{l}(x)=1 \forall l \neq i$ and $x>y$. Also, since $\psi_{l}($.$) is a distribution function, it is right continuous [5]. So$

$$
\psi_{l}(y)=1 \forall l \neq i \text {. }
$$

Suppose $y<v$. By (42):

$$
P\left\{p_{l} \in(y, v]\right\}=0, \forall l \neq i .
$$

So every price $p_{i} \in(y, v)$ is dominated by $p_{i}=v$. Hence:

$$
P\left\{p_{i} \in(y, v)\right\}=0
$$

By (43) and (44):

$$
P\left\{p_{j} \in(y, v)\right\}=0, j=1, \ldots, n .
$$

By (41), $\forall \epsilon>0, \psi_{l}(y-\epsilon)<1$ for at least one microgrid $l \neq i$; otherwise the infimum in the RHS of (41) would be less than $y$. So this microgrid $l$ plays prices just below $y$ with positive probability. Now, if microgrid $l$ sets a price $p_{l}<v$, it gets a payoff equal to the revenue, $\left(p_{l}-c\right)$, if power is sold, times the probability that power is sold. Also, by Lemma 5, $\psi_{j}(),. j=1, \ldots, n$ are continuous at all prices below $v$. So by (45), a price $p_{l}$ just below $v$ yields a higher payoff than a price just below $y$. This is because, $p_{l}-c$ is lower by approximately $v-y$ for $p_{l}$ just below $y$ than for $p_{l}$ just below $v$, but by (45) and continuity of $\psi_{j}(),. j=1, \ldots, n$, the probability that power is sold for a price $p_{l}$ just below $y$ can be made arbitrarily close to the probability that power is sold for a price $p_{l}$ just below $v$. This contradicts the assumption that microgrid $l$ plays prices just below $y$ with positive probability.

Thus, $y$ in (41) equals $v$ and hence at least one microgrid $j \neq i$ plays prices just below $v$ with positive probability. The above arguments with $j$ in place of $i$ imply that at least one microgrid other than $j$ plays prices just below $v$ with positive probability. Thus, at least two microgrids in $\{1, \ldots, n\}$ play prices just below $v$ with positive probability.

Lemma 9: $\tilde{p}=c+(v-c) \frac{1-w_{1}}{1-(1-s)^{n-1}}$.

Proof: If microgrid 1 sets the price $p_{1}=v$, then it gets an expected payoff of at least $(v-c)\left(1-w_{1}\right)$ because its power is sold at least in the event that $K_{-1}-1$ or fewer microgrids out of $2, \ldots, n$ have excess power. So $u_{1, \max } \geq(v-c)\left(1-w_{1}\right)$. Since $u_{1, \max }=(\tilde{p}-c)\left[1-(1-s)^{n-1}\right]$ by Lemma 7 , we get:

$$
\tilde{p} \geq c+(v-c) \frac{1-w_{1}}{1-(1-s)^{n-1}} .
$$

Now, by Lemma 8, at least two microgrids, say $m$ and $j$, play prices just below $v$ with positive probability. By Lemma 5, at most one of them has a jump at $v$. So assume, WLOG, that no microgrid other than $j$ has a jump at $v$. Then a price of $p_{j}=v$ is a best response for microgrid $j$ and fetches a payoff of $u_{j, \max }=(v-c)\left(1-w_{j}\right) \leq$ $(v-c)\left(1-w_{1}\right)$, where the inequality follows from (40). Since $u_{j, \max }=(\tilde{p}-c)\left[1-(1-s)^{n-1}\right]$ by Lemma 7 , we get:

$$
\tilde{p} \leq c+(v-c) \frac{1-w_{1}}{1-(1-s)^{n-1}} .
$$

The result follows from (46) and (47).

Property 2 follows from Lemmas 7 and 9.

2) Proof of Property 1 and Theorem 1: We start by proving Lemma 10, which proves most of Property 1.

Lemma 10: (i) $\phi_{2}(),. \ldots, \phi_{n}($.$) are continuous at v$. (ii) $\phi_{1}($.$) is continuous at v$ if $q_{1}=q_{2}$ and has a jump of size at most $q_{1}-q_{2}$ at $v$ if $q_{1}>q_{2}$. Also,

$$
\phi_{1}(v-) \geq q_{2} \text {. }
$$

Proof: If no microgrid $i>1$ has a jump at $v$, then microgrid 1 gets a payoff of $(v-c)\left(1-w_{1}\right)$, which equals $(\tilde{p}-c)\left[1-(1-s)^{n-1}\right]$ by Lemma 9 , for a price $p_{1}$ just below $v$ in the limit as $p_{1} \rightarrow v-$. So if a microgrid $i \geq 2$ has a jump at $v$, microgrid 1 can get a payoff strictly greater than $(\tilde{p}-$ c) $\left[1-(1-s)^{n-1}\right]$ by playing a price close enough to $v$. This contradicts the fact that $u_{1, \max }=(\tilde{p}-c)\left[1-(1-s)^{n-1}\right]$ (see Lemma 7). Thus, no microgrid $i \geq 2$ has a jump at $v$ and $\phi_{2}(),. \ldots, \phi_{n}($.$) are continuous.$

First, suppose $q_{1}=q_{2}$. If microgrid 1 has a jump at $v$, then similar to the preceding paragraph, microgrid 2 can get a payoff strictly greater than $(\tilde{p}-c)\left[1-(1-s)^{n-1}\right]$ by playing a price just below $v$, which contradicts the fact that $u_{2, \max }=$ $(\tilde{p}-c)\left[1-(1-s)^{n-1}\right]$. So $\psi_{1}($.$) is continuous.$

Now suppose $q_{1}>q_{2}$. First, suppose microgrid 1 has a jump of size exactly $q_{1}-q_{2}$ at $v$. Then if microgrid 2 sets a price just below $v$, then the probability of being undercut by microgrid $j \in\{3, \ldots, n\}$ is approximately $q_{j}$. Also, since microgrid 1 has a jump of size $q_{1}-q_{2}$ at $v$, the probability of being undercut by microgrid 1 is approximately $q_{1}-\left(q_{1}-q_{2}\right)=q_{2}$. So at a price just below $v$, microgrid 2 sees the same set of probabilities of being undercut by microgrids other than itself as microgrid 1 would see if it set a price just below $v$. Hence, 
by the first paragraph of this proof, microgrid 2 gets a payoff of approximately $(\tilde{p}-c)\left[1-(1-s)^{n-1}\right]$ at a price just below $v$.

Hence, if microgrid 1 has a jump of size, not equal to, but greater than $q_{1}-q_{2}$ at $v$, microgrid 2 gets a payoff of strictly greater than $(\tilde{p}-c)\left[1-(1-s)^{n-1}\right]$ at a price just below $v$. This contradicts the fact that $u_{2, \max }=(\tilde{p}-$ c) $\left[1-(1-s)^{n-1}\right]$.

Thus, microgrid 1 has a jump of at most size $q_{1}-q_{2}$ at $v$. So $\phi_{1}(v)-\phi_{1}(v-) \leq q_{1}-q_{2}$. This, along with $\phi_{1}(v)=q_{1}$, gives (48).

Given Lemmas 5 and 10, Property 1 follows once we show that the jump of $\phi_{1}($.$) at v$ is exactly $q_{1}-q_{2}$. We will prove this in Lemma 15, which we will prove after proving Part 2 of Theorem 1 in Lemma 14.

Let $F_{-i}(x)$ be as in Definition 2. The following lemma will be used later.

Lemma 11: For a fixed $x \in(\tilde{p}, v]$, and microgrids $i$ and $j$, (i) $F_{-i}(x)=F_{-j}(x)$ iff $\phi_{i}(x)=\phi_{j}(x)$, (ii) $F_{-i}(x)<F_{-j}(x)$ iff $\phi_{i}(x)>\phi_{j}(x)$.

Proof: Let $K_{-(i, j)}$ be the number of microgrids out of $\{1, \ldots, n\} \backslash\{i, j\}$ that have 1 unit of deficit power. Let $p_{(l)}^{\prime}$ be the $l$ 'th smallest pseudo-price out of the pseudo-prices of microgrids $\{1, \ldots, n\} \backslash\{i, j\}$ (with $p_{(l)}^{\prime}$ defined to be 0 if $l \leq 0$ and $v+1$ if $l>n-2$ ). Now, microgrid $j$ has (i) 1 unit of deficit power w.p. $s$, (ii) neither excess nor deficit power w.p. $1-q_{j}-s$, (iii) 1 unit of excess power and $p_{j} \leq x$ w.p. $q_{j} \psi_{j}(x)$ and (iv) 1 unit of excess power and $p_{j}>x$ w.p. $q_{j}\left(1-\psi_{j}(x)\right)$. Conditioning on the preceding four events, we get:

$$
\begin{aligned}
& F_{-i}(x) \\
& =P\left\{p_{-i}^{\prime} \leq x\right\} \\
& =s P\left\{p_{\left(K_{-(i, j)}+1\right)}^{\prime} \leq x\right\}+\left(1-q_{j}-s\right) P\left\{p_{\left(K_{-(i, j)}\right)}^{\prime} \leq x\right\} \\
& +q_{j} \psi_{j}(x) P\left\{p_{\left(K_{-(i, j)}^{\prime}-1\right)}^{\prime} \leq x\right\} \\
& +q_{j}\left(1-\psi_{j}(x)\right) P\left\{p_{\left(K_{-(i, j)}\right)}^{\prime} \leq x\right\} \\
& =s P\left\{p_{\left(K_{-(i, j)}+1\right)}^{\prime} \leq x\right\}+(1-s) P\left\{p_{\left(K_{-(i, j)}\right)}^{\prime} \leq x\right\} \\
& +\phi_{j}(x)\left[P\left\{p_{\left(K_{-(i, j)}-1\right)}^{\prime} \leq x\right\}-P\left\{p_{\left(K_{-(i, j)}^{\prime}\right)}^{\prime} \leq x\right\}\right\}_{49}
\end{aligned}
$$

Similarly:

$$
\begin{aligned}
& F_{-j}(x) \\
& \quad=\quad s P\left\{p_{\left(K_{-(i, j)}^{\prime}+1\right)}^{\prime} \leq x\right\}+(1-s) P\left\{p_{\left(K_{-(i, j)}\right)}^{\prime} \leq x\right\} \\
& \quad+\phi_{i}(x)\left[P\left\{p_{\left(K_{-(i, j)}-1\right)}^{\prime} \leq x\right\}-P\left\{p_{\left(K_{-(i, j)}\right)}^{\prime} \leq x(5)\right] 0\right)
\end{aligned}
$$

Subtracting (50) from (49), we get:

$$
\begin{aligned}
& F_{-i}(x)-F_{-j}(x)=\left(\phi_{j}(x)-\phi_{i}(x)\right) \times \\
& {\left[P\left\{p_{\left(K_{-(i, j)}-1\right)}^{\prime} \leq x\right\}-P\left\{p_{\left(K_{-(i, j)}\right)}^{\prime} \leq x\right\}\right]}
\end{aligned}
$$

Now, since $x>\tilde{p}$, all microgrids play prices in $(\tilde{p}, x)$ with positive probability by Lemma 7. So:

$$
\phi_{l}(x)=P\left\{p_{l}^{\prime} \leq x\right\}>0, l=1, \ldots, n .
$$

Also,

$$
\phi_{l}(x) \leq \phi_{l}(v)=q_{l}<1, l=1, \ldots, n .
$$

By (52) and (53):

$$
0<\phi_{l}(x)<1, l=1, \ldots, n .
$$

Also, $P\left\{p_{\left(K_{-(i, j)}-1\right)}^{\prime} \leq x\right\}-P\left\{p_{\left(K_{-(i, j)}\right)}^{\prime} \leq x\right\}$ is the probability of the event that exactly $K_{-(i, j)}-1$ pseudo-prices out of the pseudo-prices of the microgrids $\{1, \ldots, n\} \backslash\{i, j\}$ are $\leq x$, which happens in particular when $K_{-(i, j)}=1$ and no pseudo-price out of $\{1, \ldots, n\} \backslash\{i, j\}$ is $\leq x$. By (54), the probability of the latter event is positive and hence $P\left\{p_{\left(K_{-(i, j)}-1\right)}^{\prime} \leq x\right\}-P\left\{p_{\left(K_{-(i, j)}\right)}^{\prime} \leq x\right\}>0$. The result now follows from (51).

Now, in a sequence of two lemmas, we prove that each microgrid plays prices in every sub-interval of its support set with positive probability- a result that will be used to prove part 2 of Theorem 1. The following lemma generalizes Lemma 8.

Lemma 12: Let $\tilde{p} \leq a<b \leq v$. Then at least two microgrids play prices in $(a, b)$ with positive probability.

Proof: If $b=v$, then the claim is true by Lemma 8. If $a=\tilde{p}$, then the claim is true by Lemma 5 and Lemma 7, since $\tilde{p}<v$ is the lower endpoint of the support set of all microgrids and no microgrid has a jump at $\tilde{p}$; hence all microgrids play prices just above $\tilde{p}$ with positive probability.

Now, fix any $a, b$ such that $\tilde{p}<a<b<v$. Let:

$$
\underline{a}=\inf \left\{x \leq a: \psi_{j}(x)=\psi_{j}(a) \forall j=1, \ldots, n\right\}
$$

By Lemma 7, $\underline{a}>\tilde{p}$. Also, by definition of $\underline{a}, P\left\{p_{j} \in\right.$ $[\underline{a}, a]\}=0 \forall j=1, \ldots, n$.

By definition of $\underline{a}$, at least one microgrid, say microgrid $i$, plays prices just below $\underline{a}$ with positive probability. (If not, then the infimum in (55) would be less than $\underline{a}$.) This implies that at least one microgrid $j \neq i$ plays prices in $(\underline{a}, b)$ with positive probability. (If not, then $p_{i}=b$ would yield a strictly higher payoff to microgrid $i$ than prices just below $\underline{a}$.) Now, if microgrid $j$ is the only microgrid among microgrids $\{1, \ldots, n\}$ who play prices in $(\underline{a}, b)$ with positive probability, then $p_{j}=b$ yields a strictly higher payoff than $p_{j} \in(\underline{a}, b)$, which is a contradiction. So at least two microgrids play prices in $(\underline{a}, b)$ with positive probability. But $P\left\{p_{l} \in[\underline{a}, a]\right\}=0 \forall l=1, \ldots, n$ by definition of $\underline{a}$. Hence, at least two microgrids play prices in $(a, b)$ with positive probability.

Lemma 13: If $\tilde{p} \leq x<y<v$ and $\psi_{i}(x)=\psi_{i}(y)$ for some microgrid $i$, then $\psi_{i}(v-)=\psi_{i}(x)$.

Thus, if $x \geq \tilde{p}$ is the left endpoint of an interval of constancy of $\psi_{i}($.$) for some i$, then to the right of $x$, the interval of constancy extends at least until $v$ (there may be a jump at $v$ ).

Proof: Suppose not, i.e.:

$$
\psi_{i}(v-)>\psi_{i}(x) \text {. }
$$

Let:

$$
\bar{y}=\sup \left\{z \geq x: \psi_{i}(z)=\psi_{i}(x)\right\}
$$

By (56) and (57), we get $\bar{y}<v$. So by Lemma 5 , no microgrid among $\{1, \ldots, n\} \backslash i$ has a jump at $\bar{y}$. Also, microgrid $i$ uses prices just above $\bar{y}$ with positive probability (if not, the supremum in the RHS of (57) would be $>\bar{y}$ ). So $\bar{y}$ is a best response for microgrid $i$ and hence:

$$
\begin{aligned}
E\left\{u_{i}\left(\bar{y}, \psi_{-i}\right)\right\} & =(\bar{y}-c)\left(1-F_{-i}(\bar{y})\right) \\
& =u_{i, \max }=(\tilde{p}-c)\left[1-(1-s)^{n-1}\right](5,8)
\end{aligned}
$$

where the last equality follows from Lemma 7.

Now, by Lemma 12 , there exists a microgrid $j \neq i$ who plays prices just below $\bar{y}$ with positive probability. Since no 
microgrid among $\{1, \ldots, n\} \backslash j$ has a jump at $\bar{y}, \bar{y}$ is a best response for microgrid $j$. Hence:

$$
\begin{aligned}
E\left\{u_{j}\left(\bar{y}, \psi_{-j}\right)\right\} & =(\bar{y}-c)\left(1-F_{j}(\bar{y})\right) \\
& =u_{j, \max }=(\tilde{p}-c)\left[1-(1-s)^{n-1}(5.9)\right.
\end{aligned}
$$

By (58) and (59), $F_{-i}(\bar{y})=F_{-j}(\bar{y})$. So by Lemma 11 :

$$
\phi_{i}(\bar{y})=\phi_{j}(\bar{y}) \text {. }
$$

But since microgrid $j$ plays prices just below $\bar{y}$ with positive probability, there exists $\epsilon>0$ such that $x<\bar{y}-\epsilon$ and $\bar{y}-\epsilon$ is a best response for microgrid $j$. So

$$
\phi_{j}(\bar{y}-\epsilon)<\phi_{j}(\bar{y}) .
$$

But by (57) and the continuity of $\phi_{i}($.$) at \bar{y}$ :

$$
\phi_{i}(\bar{y})=\phi_{i}(\bar{y}-\epsilon) .
$$

By (60), (61) and (62), $\phi_{i}(\bar{y}-\epsilon)>\phi_{j}(\bar{y}-\epsilon)$. So by Lemma 11:

$$
F_{-j}(\bar{y}-\epsilon)>F_{-i}(\bar{y}-\epsilon)
$$

This implies:

$$
\begin{aligned}
(\tilde{p}-c)\left[1-(1-s)^{n-1}\right] & =E\left\{u_{j}\left(\bar{y}-\epsilon, \psi_{-j}\right)\right\} \\
& =(\bar{y}-\epsilon-c)\left(1-F_{-j}(\bar{y}-\epsilon)\right) \\
& <(\bar{y}-\epsilon-c)\left(1-F_{-i}(\bar{y}-\epsilon)\right) \\
& =E\left\{u_{i}\left(\bar{y}-\epsilon, \psi_{-i}\right)\right\}
\end{aligned}
$$

which contradicts the fact that every microgrid gets a payoff of $(\tilde{p}-c)\left[1-(1-s)^{n-1}\right]$ at a best response in the NE.

Lemma 14: Part 2 of Theorem 1 holds.

Proof: We prove the result by induction. Let:

$$
R_{n}=\inf \left\{x \geq \tilde{p}: \exists y>x \text { and } i \text { s.t. } \phi_{i}(y)=\phi_{i}(x)\right\}
$$

Note that $R_{n}$ is the smallest value $\geq \tilde{p}$ that is the left endpoint of an interval of constancy for some $\phi_{i}($.$) . For this i, \phi_{i}\left(R_{n}\right)=$ $\phi_{i}(y)$ for some $y>R_{n}{ }^{10}$. We must have $R_{n}>\tilde{p}$. This is because, if $R_{n}=\tilde{p}$, then $\phi_{i}(y)=\phi_{i}(\tilde{p})$. But $\phi_{i}(\tilde{p})=0$, since $\tilde{p}$ is the lower endpoint of the support set of $\phi_{i}($.$) by Lemma 7$. So $\phi_{i}(y)=0$, which implies that the lower endpoint of the support set of $\phi_{i}($.$) is \geq y>\tilde{p}$. This contradicts Lemma 7 . Thus, $R_{n}>\tilde{p}$.

Now, by definition of $R_{n}$, all microgrids play every subinterval in $\left[\tilde{p}, R_{n}\right)$ with positive probability and hence every price $x \in\left[\tilde{p}, R_{n}\right)$ is a best response for every microgrid. So similar to the derivation of (9), for $j \in\{1, \ldots, n\}$ and $x \in\left[\tilde{p}, R_{n}\right), E\left\{u_{j}\left(x, \psi_{-j}\right)\right\}=(x-c)\left(1-F_{-j}(x)\right)=$ $(\tilde{p}-c)\left[1-(1-s)^{n-1}\right]$. Hence, $F_{-1}(x)=\ldots=F_{-n}(x)$ and by Lemma 11 ,

$$
\phi_{1}(x)=\ldots=\phi_{n}(x)=\phi(x) \text { (say), } \tilde{p} \leq x<R_{n} .
$$

which proves (6) for $j=n$.

Case (i): Suppose $R_{n}=v$. Then $\phi_{l}\left(R_{n}\right)=q_{l}, l=1, \ldots, n$ (since $\psi_{l}(v)=1$ ), which proves (7).

Case (ii): Now suppose $R_{n}<v$. Then $\phi_{j}(),. j=1, \ldots, n$ are continuous at $R_{n}$ by Lemma 5 . So by (64):

$$
\phi_{1}\left(R_{n}\right)=\phi_{2}\left(R_{n}\right)=\ldots=\phi_{n}\left(R_{n}\right) .
$$

${ }^{10}$ Note that $\phi_{i}($.$) is a distribution function and hence is right continuous [5]$ So $\phi_{i}\left(R_{n}+\right)=\phi_{i}\left(R_{n}\right)$.
Since $R_{n}$ is the left endpoint of an interval of constancy of $\phi_{i}($.$) , by Lemma 13$ :

$$
\phi_{i}\left(R_{n}\right)=\phi_{i}(v-)=\phi_{n}\left(R_{n}\right) \leq q_{n}
$$

where the second equality follows from (65).

Now, suppose $i=1$. Then by (48) and (66):

$$
\phi_{i}\left(R_{n}\right) \geq q_{2} \text {. }
$$

By (66), (67) and (1), $q_{2}=q_{3}=\ldots=q_{n}=\phi_{i}\left(R_{n}\right)$. Also, by $(65), \phi_{j}\left(R_{n}\right)=q_{j}, j=2, \ldots, n$. So $\psi_{j}\left(R_{n}\right)=1, j=$ $2, \ldots, n$. This implies, since $R_{n}<v$ by assumption, that at most one microgrid (microgrid 1) plays prices in the interval $\left(R_{n}, v\right)$ with positive probability, which contradicts Lemma 8. Thus, $i \neq 1$.

So by Lemma $10, \phi_{i}($.$) is continuous at v$ and $\phi_{i}(v-)=$ $\phi_{i}(v)=q_{i}$. So by (66):

$$
\phi_{i}\left(R_{n}\right)=q_{i} .
$$

By (65) and (68), $\phi_{n}\left(R_{n}\right)=q_{i}$. If $q_{i}>q_{n}$, then $\phi_{n}\left(R_{n}\right)>q_{n}$, which is a contradiction because $\phi_{n}\left(R_{n}\right)=q_{n} \psi_{n}\left(R_{n}\right) \leq q_{n}$. So $q_{i} \leq q_{n}$. Also, since $q_{i} \geq q_{n}$ by (1), $q_{i}=q_{n}$. So:

$$
\phi_{n}\left(R_{n}\right)=q_{n} .
$$

which proves (7) for $j=n$.

Now, as induction hypothesis, suppose there exist thresholds:

$$
\tilde{p}<R_{n} \leq R_{n-1} \leq \ldots \leq R_{i+1} \leq v
$$

such that for each $j \in\{i+1, \ldots, n\}, \phi_{j}\left(R_{j}\right)=q_{j}$,

$$
\phi_{1}(x)=\ldots=\phi_{j}(x)=\phi(x), \tilde{p} \leq x<R_{j},
$$

and each of microgrids $1, \ldots, j$ plays every sub-interval in $\left[\tilde{p}, R_{j}\right)$ with positive probability.

First, suppose $R_{i+1}<v$. Let:

$$
\begin{aligned}
R_{i}= & \inf \left\{x \geq R_{i+1}: \exists y>x \text { and } j \in\{1, \ldots, i\}\right. \\
& \text { s.t. } \left.\phi_{j}(y)=\phi_{j}(x)\right\} .
\end{aligned}
$$

If $R_{i}=R_{i+1}$, then clearly by (70):

$$
\phi_{1}(x)=\ldots=\phi_{i}(x)=\phi(x), \tilde{p} \leq x<R_{i}
$$

which proves (6) for $j=i$. Also, similar to (69), it can be shown that $\phi_{i}\left(R_{i}\right)=q_{i}$, which proves (7) for $j=i$ and completes the inductive step. Now suppose $R_{i}>R_{i+1}$. Then similar to the proof of (64), it can be shown that:

$$
\phi_{1}(x)=\ldots=\phi_{i}(x)=\phi(x), R_{i+1} \leq x<R_{i} .
$$

By (70) and (72):

$$
\phi_{1}(x)=\ldots=\phi_{i}(x)=\phi(x), \tilde{p} \leq x<R_{i} .
$$

which proves (6) for $j=i$. Also, similar to the proof of (69), it can be shown that $\phi_{i}\left(R_{i}\right)=q_{i}$, which proves (7) for $j=i$. This completes the induction.

If $R_{i+1}=v$, then the induction is completed by simply setting $R_{1}=\ldots=R_{i}=v$.

It remains to show that $R_{1}=R_{2}=v$. If $R_{1}<v$, then no microgrid plays a price in $\left(R_{1}, v\right)$, which contradicts Lemma 8. So $R_{1}=v$. If $R_{2}<v$, then only microgrid 1 plays prices in $\left(R_{2}, v\right)$ with positive probability, which again contradicts Lemma 8. So $R_{2}=v$. 
Now, Lemma 10 showed that if $q_{1}>q_{2}$, then $\phi_{1}($.$) has a$ jump of size at most $q_{1}-q_{2}$ at $v$. The following lemma shows that the size of the jump is in fact exactly $q_{1}-q_{2}$.

Lemma 15: If $q_{1}>q_{2}$, then $\phi_{1}($.$) has a jump of size q_{1}-q_{2}$ at $v$. So:

Proof: By Lemma 14, $\phi_{1}(x)=\phi_{2}(x)$ for all $x<R_{2}=v$.

$$
\begin{aligned}
\phi_{1}(v-) & =\phi_{2}(v-) \\
& =\phi_{2}(v) \quad\left(\text { since } \phi_{2}(.) \text { is continuous by Lemma } 10\right) \\
& =q_{2}
\end{aligned}
$$

Also, $\phi_{1}(v)=q_{1} \psi_{1}(v)=q_{1}$. So $\phi_{1}(v)-\phi_{1}(v-)=q_{1}-q_{2}$.

Finally, (i) Property 1 follows from Lemmas 5, 10 and 15; and (ii) Theorem 1 follows from Properties 1 and 2 and Lemma 14.

\section{B. Proofs of results in Section III-B}

Proof of Lemma 1: Since each of the $n-1$ events in the definition of $f_{i}(y)$ results in deficit w.p. $s$, we get:

$$
P\left(K_{-1}=k\right)=\left(\begin{array}{c}
n-1 \\
k
\end{array}\right) s^{k}(1-s)^{n-1-k} .
$$

Let $v_{k, l_{1}, l_{2}}\left(q_{i+1}, \ldots, q_{n}, s\right)$ be the probability that out of the $n-i$ events with success probabilities $q_{i+1}, \ldots, q_{n}$ in the definition of $f_{i}($.$) , exactly l_{1}$ result in deficit and $l_{2}$ result in success given that $K_{-1}=k$. Also, let $h_{k, l_{1}, l_{2}}(y)$ be the probability that out the $i-1$ events with success probability $y$ each in the definition of $f_{i}(),. k-l_{2}$ or more result in success given that exactly $k-l_{1}$ result in deficit. Now, given that exactly $k-l_{1}$ events result in deficit, the remaining $(i-1)-\left(k-l_{1}\right)$ events do not result in deficit, and hence the probability that each of these results in success is $\frac{y}{1-s}$. So:

$$
\begin{aligned}
h_{k, l_{1}, l_{2}}(y)= & \sum_{l_{3}=k-l_{2}}^{i-1-k+l_{1}}\left(\begin{array}{c}
i-1-k+l_{1} \\
l_{3}
\end{array}\right)\left(\frac{y}{1-s}\right)^{l_{3}} \times \\
& \left(1-\frac{y}{1-s}\right)^{i-1-k+l_{1}-l_{3}}
\end{aligned}
$$

Also:

$$
\begin{aligned}
& f_{i}(y)= \sum_{k, l_{1}, l_{2}} P\left(K_{-1}=k\right) v_{k, l_{1}, l_{2}}\left(q_{i+1}, \ldots, q_{n}, s\right) h_{k, l_{1}, l_{2}}(y) \\
&= \sum_{k, l_{1}, l_{2}}\left(\begin{array}{c}
n-1 \\
k
\end{array}\right) s^{k}(1-s)^{n-1-k} \times \\
& v_{k, l_{1}, l_{2}}\left(q_{i+1}, \ldots, q_{n}, s\right) h_{k, l_{1}, l_{2}}(y) .
\end{aligned}
$$

where the second step follows from (73). Now, $h_{k, l_{1}, l_{2}}($.$) in$ (74) is a polynomial function of $y$ and hence continuous in $y$. Also, it is a strictly increasing function of $y$ [9]. So by (75), $f_{i}(y)$ is a strictly increasing and continuous function of $y$.

Proof of Lemma 2: It can be checked from the definition of the function $f_{i}($.$) (see Definition 3) that:$

$$
f_{i}\left(q_{i+1}\right)=f_{i+1}\left(q_{i+1}\right) .
$$

Also, replacing $i$ with $i+1$ in (13), we get:

$$
f_{i+1}\left(q_{i+1}\right)=g\left(R_{i+1}\right) \text {. }
$$

By (76) and (77), we get:

$$
f_{i}\left(q_{i+1}\right)=g\left(R_{i+1}\right) .
$$

Now, by Lemma $1, f_{i}($.$) is invertible. By (18), \phi($.$) is unique$ and is given by:

$$
\phi(x)=f_{i}^{-1}(g(x)), R_{i+1} \leq x<R_{i} .
$$

Also, by (78) and (13), $f_{i}\left(q_{i+1}\right)=g\left(R_{i+1}\right)$ and $f_{i}\left(q_{i}\right)=$ $g\left(R_{i}\right)$. So $f_{i}($.$) is a continuous one-to-one map from the$ compact set $\left[q_{i+1}, q_{i}\right]$ onto $\left[g\left(R_{i+1}\right), g\left(R_{i}\right)\right]$, and hence $f_{i}^{-1}($. is continuous (see Theorem 4.17 in [6]). Also, $g(x)$ in (11) is continuous for all $x \in[\tilde{p}, v)$ since $x \geq \tilde{p}>c$. So from (79), $\phi($.$) is a continuous function on \left[R_{i+1}, R_{i}\right]$, since it is the composition of continuous functions $f_{i}^{-1}$ and $g$ (see Theorem 4.7 in [6]). Also, by Lemma 1, $f_{i}($.$) is strictly increasing; so$ $f_{i}^{-1}($.$) is strictly increasing. Also, it follows from (11) that$ $g($.$) is strictly increasing. By (79), \phi($.$) is the composition$ of the strictly increasing functions $f_{i}^{-1}($.$) and g($.$) and hence$ is strictly increasing on $\left[R_{i+1}, R_{i}\right]$. Also, by (78) and (79), $\phi\left(R_{i}\right)=f_{i}^{-1}\left(g\left(R_{i}\right)\right)=q_{i}$.

Thus, the function $\phi($.$) is strictly increasing and continuous$ within each individual interval $\left[R_{i+1}, R_{i}\right]$; also, $\phi\left(R_{i}\right)=q_{i}$, $i=2, \ldots, n$, and hence $\phi($.$) is continuous at the endpoints$ $R_{i}, i=2, \ldots, n$ of these intervals. So $\phi($.$) is strictly increasing$ and continuous on $[\tilde{p}, v)$.

It remains to show that $\phi(\tilde{p})=0$. By definition of the function $f_{i}(),. f_{n}(0)=(1-s)^{n-1}$. As shown above, $f_{n}($.$) is$ one-to-one. So $f_{n}^{-1}\left((1-s)^{n-1}\right)=0$. Also, by (11), $g(\tilde{p})=$ $(1-s)^{n-1}$; also, recall that $R_{n+1}=\tilde{p}$. Putting $i=n$ and $x=R_{n+1}=\tilde{p}$ in (79), we get $\phi(\tilde{p})=f_{n}^{-1}(g(\tilde{p}))=f_{n}^{-1}((1-$ $\left.s)^{n-1}\right)=0$.

Proof of Theorem 2: By Lemma 2 and equation (8), the functions $\phi_{i}(),. i=1, \ldots, n$ computed in Section III-B are continuous and non-decreasing on $[\tilde{p}, v]$; also, $\phi_{i}(\tilde{p})=0$ and $\phi_{i}(v)=q_{i}$. This is consistent with the fact that $\phi_{i}($.$) is the$ d.f. of the pseudo-price $p_{i}^{\prime}$ and hence should be non-decreasing and right continuous [5], and $\phi_{i}(v)=q_{i} \psi_{i}(v)=q_{i}$ (see the beginning of Section III).

Now, we have shown in Sections III-A and III-B that (8) is a necessary condition for the functions $\phi_{i}(),. i=1, \ldots, n$ to constitute a NE. We now show sufficiency. Suppose for each $i \in\{1, \ldots, n\}$, microgrid $i$ uses the strategy $\phi_{i}($.$) in$ (8). Similar to the derivation of (9), the expected payoff that microgrid $i$ gets at a price $x \in[\tilde{p}, v)$ is:

$$
E\left\{u_{i}\left(x, \psi_{-i}\right)\right\}=(x-c)\left(1-F_{-i}(x)\right) .
$$

Now, for $x \in\left[\tilde{p}, R_{i}\right)$, by (8), $\phi_{i}(x)=\phi_{1}(x)=\phi(x)$, and hence by Lemma 11, $F_{-i}(x)=F_{-1}(x)$. By (9), (80) and the fact that $F_{-i}(x)=F_{-1}(x)$, for microgrid $i$, prices $x \in\left[\tilde{p}, R_{i}\right)$ fetch an expected payoff of $(\tilde{p}-c)\left[1-(1-s)^{n-1}\right]$.

Now let $x \in\left[R_{i}, v\right)$. Note that $R_{i} \leq x<v=R_{1}$. So by $(8), \phi_{i}(x)=q_{i}$ and $\phi_{1}(x)=\phi(x) \geq \phi\left(R_{i}\right)=q_{i}$ by Lemma 2. So $\phi_{1}(x) \geq \phi_{i}(x)$. Hence, by Lemma $11, F_{-1}(x) \leq$ $F_{-i}(x)$, which by (9) and (80) implies $E\left\{u_{i}\left(x, \psi_{-i}\right)\right\} \leq(\tilde{p}-$ c) $\left[1-(1-s)^{n-1}\right]$.

Finally, note that a price below $\tilde{p}$ fetches a payoff of less than $(\tilde{p}-c)\left[1-(1-s)^{n-1}\right]$ for microgrid $i$. So each price in $\left[\tilde{p}, R_{i}\right)$ is a best response for microgrid $i$; also, by (8), it randomizes over prices only in this range under $\phi_{i}($.$) . So$ $\phi_{i}($.$) is a best response. Thus, the functions \phi_{i}(),. i=1, \ldots, n$ constitute a NE. 


\section{Proofs of results in Section IV}

Proof of Theorem 3: First, we show that the functions $\psi_{1}(),. \psi_{2}($.$) and \psi_{3}($.$) in (24), (25) and (26) are valid d.f.s.$ It can be easily checked that $\psi_{2}($.$) and \psi_{3}($.$) are continuous$ everywhere and $\psi_{1}($.$) is continuous everywhere except possi-$ bly at $v$. Also, $\psi_{1}(v-) \leq 1$ iff (20) holds, which is true by assumption. If $\psi_{1}(v-)<1$, then $\psi_{1}($.$) has a jump at v$. Since $\psi_{1}(x)=1$ for $x \geq v, \psi_{1}($.$) is right continuous at v$. Also, with $F($.$) as in (23), F^{\prime}(x)=\frac{\tilde{p}-c}{(x-c)^{2}}>0$ for $x \in[\tilde{p}, v]$ and hence $F($.$) is strictly increasing on [\tilde{p}, v]$. So by $(24),(25)$ and (26), $\psi_{1}(),. \psi_{2}($.$) and \psi_{3}($.$) are non-decreasing. Thus, \psi_{1}($.$) ,$ $\psi_{2}($.$) and \psi_{3}($.$) are non-decreasing and right continuous, and$ hence are valid d.f.s [5].

Now, note that under the strategies $\psi_{1}(),. \psi_{2}($.$) and \psi_{3}($.$) in$ (24), (25) and (26), microgrid 3 (respectively, microgrids 1 and 2 ) play every sub-interval in the range $\left[\tilde{p}, R_{3}\right.$ ) (respectively, $[\tilde{p}, v)$ ) with positive probability and microgrid 1 can have a jump at $v$. The microgrids do not set prices other than these. In the rest of the proof, we will show that microgrid 1 (respectively, 2,3 ) gets an expected payoff of $u_{1, \max }$ (respectively, $u_{2, \max }, u_{3, \max }$ ) at a price $x \in[\tilde{p}, v]$ (respectively, $x \in[\tilde{p}, v), x \in\left[\tilde{p}, R_{3}\right)$ ) and a payoff less than or equal to $u_{1, \max }$ (respectively, $u_{2, \max }, u_{3, \max }$ ) at every other price. It will follow that in the strategy profile in (24), (25) and (26), every microgrid randomizes only over best responses and hence it is a NE.

Now, if no microgrid out of microgrids 2 and 3 has a jump at price $x$ and microgrid 1 sets the price $x$, then its power is sold (i) if both of microgrids 2 and 3 have deficit power, (ii) one of them has deficit power and the other has neither excess nor deficit or (iii) one of them has deficit power, the other has excess power and sets a price greater than $x^{11}$. So:

$$
\begin{aligned}
E\left\{u_{1}\left(x, \psi_{-1}\right)\right\} & \\
= & (x-c)\left\{s_{2} s_{3}+s_{2}\left(1-s_{3}-q_{3}\right)+s_{3}\left(1-s_{2}-q_{2}\right)\right. \\
& \left.\quad+s_{2} q_{3}\left(1-\psi_{3}(x)\right)+s_{3} q_{2}\left(1-\psi_{2}(x)\right)\right\} \\
= & (x-c)\left(s_{2}+s_{3}-s_{2} s_{3}-s_{2} \phi_{3}(x)-s_{3} \phi_{2}(x)\right)
\end{aligned}
$$

Similarly,

$$
\begin{aligned}
& E\left\{u_{2}(x, \psi-2)\right\} \\
& \quad=(x-c)\left(s_{1}+s_{3}-s_{1} s_{3}-s_{1} \phi_{3}(x)-s_{3} \phi_{1}(x)\right)(82)
\end{aligned}
$$

and

$$
\begin{aligned}
& E\left\{u_{3}\left(x, \psi_{-3}\right)\right\} \\
& \quad=(x-c)\left(s_{1}+s_{2}-s_{1} s_{2}-s_{1} \phi_{2}(x)-s_{2} \phi_{1}(x)\right)(83)
\end{aligned}
$$

Using (81), (82) and (83), $\psi_{1}(),. \psi_{2}($.$) and \psi_{3}($.$) from$ (24), (25) and (26) and the fact that $\phi_{i}(x)=q_{i} \psi_{i}(x)$, $i=1,2,3$, we get $E\left\{u_{1}\left(x, \psi_{-1}\right)\right\}=u_{1, \max }$ for $x \in$ $[\tilde{p}, v], E\left\{u_{2}\left(x, \psi_{-2}\right)\right\}=u_{2, \max }$ for $x \in[\tilde{p}, v)$ and $E\left\{u_{3}\left(x, \psi_{-3}\right)\right\}=u_{3, \max }$ for $x \in\left[\tilde{p}, R_{3}\right)$, where $u_{1, \max }$, $u_{2, \max }$ and $u_{3, \max }$ are as in (27), (28) and (29).

Next, we show that microgrid 3's expected payoff at a price $x \in\left(R_{3}, v\right)$ is $\leq u_{3, \max }$. The value of $\phi_{1}(x)$ is given by (24)

\footnotetext{
${ }^{11}$ Note that microgrid 1's power can also be sold if one or both of microgrids 2 and 3 set the price $x$, but the probability of this event is 0 by assumption.
}

along with the fact that $\phi_{1}(x)=q_{1} \psi_{1}(x)$. So:

$$
\phi_{1}(x)=\frac{1}{s_{3}}\left\{\left(s_{1}+s_{3}-s_{1} s_{3}\right) F(x)-s_{1} q_{3}\right\}, x \in\left(R_{3}, v\right)
$$

Let:

$$
\tilde{\phi}_{1}(x)=\left(1-\frac{s_{1}}{2}\right) F(x), x \in\left(R_{3}, v\right)
$$

By (84) and (85), on $x \in\left(R_{3}, v\right)$ :

$$
\phi_{1}(x)-\tilde{\phi}_{1}(x)=s_{1}\left\{\left(\frac{1}{s_{3}}-\frac{1}{2}\right) F(x)-\frac{q_{3}}{s_{3}}\right\} .
$$

Now, $\frac{1}{q_{3}}\left(1-\frac{s_{3}}{2}\right) F(x) \geq 1$ because $\frac{1}{q_{3}}\left(1-\frac{s_{3}}{2}\right) F\left(R_{3}\right)=$ $\psi_{3}\left(R_{3}\right)=1$ (by (26) and the continuity of $\psi_{3}($.$) ), x \geq R_{3}$ and $F($.$) in (23) is an increasing function of x$. So by (86):

$$
\phi_{1}(x) \geq \tilde{\phi}_{1}(x), x \in\left(R_{3}, v\right) .
$$

Similarly:

$$
\phi_{2}(x) \geq \tilde{\phi}_{2}(x), x \in\left(R_{3}, v\right)
$$

where:

$$
\tilde{\phi}_{2}(x)=\left(1-\frac{s_{2}}{2}\right) F(x), x \in\left(R_{3}, v\right)
$$

Now, for $x \in\left(R_{3}, v\right)$, by (83):

$$
\begin{aligned}
E\{ & \left.u_{3}\left(x, \psi_{-3}\right)\right\} \\
= & (x-c)\left[s_{1}+s_{2}-s_{1} s_{2}-s_{2} \phi_{1}(x)-s_{1} \phi_{2}(x)\right] \\
\leq & (x-c)\left[s_{1}+s_{2}-s_{1} s_{2}-s_{2} \tilde{\phi}_{1}(x)-s_{1} \tilde{\phi}_{2}(x)\right] \\
& (\text { by }(87) \text { and (88)) } \\
= & (\tilde{p}-c)\left(s_{1}+s_{2}-s_{1} s_{2}\right)(\text { by (85) and (89)) } \\
= & u_{3, \max }(\text { by (29)) }
\end{aligned}
$$

Thus, the expected payoff of microgrid 3 is $\leq u_{3, \max }$ for prices in $\left(R_{3}, v\right)$. The expected payoff at $v$ is also $\leq u_{3, \max }$ because the payoff at every $x<v$ is $\leq u_{3, \max }$ and microgrid 1 possibly has a jump at $v$.

As shown above, for microgrid 2, the expected payoff at every price in $(\tilde{p}, v)$ is $u_{2, \max }$ and the expected payoff at $v$ is $\leq u_{2, \max }$ since microgrid 1 possibly has a jump at $v$. Also, for microgrid 1 , the expected payoff at every price in $[\tilde{p}, v]$ equals $u_{1, \max }$.

Finally, by (81), (25), (26) and the fact that $\phi_{i}(x)=$ $q_{i} \psi_{i}(x), i=2,3$, at a price $x<\tilde{p}, E\left\{u_{1}\left(x, \psi_{-1}\right)\right\}=$ $(x-c)\left(s_{2}+s_{3}-s_{2} s_{3}\right)<(\tilde{p}-c)\left(s_{2}+s_{3}-s_{2} s_{3}\right)=u_{1, \max }$ and similarly $E\left\{u_{2}\left(x, \psi_{-2}\right)\right\}<u_{2, \max }$ and $E\left\{u_{3}\left(x, \psi_{-3}\right)\right\}<$ $u_{3, \max }$.

The result follows.

\section{Proofs of results in Section V}

The proofs are similar to the proofs (in Appendices A and B) of the results in Section III, as we now explain. Throughout, $v$ in Appendices $\mathrm{A}$ and $\mathrm{B}$ is replaced with $v_{T}$.

First, we show that Properties 1 and 2 and Theorem 1 hold with the changes stated in Section V. Lemmas 5, 8, 6 and their proofs hold in the present context without change.

Lemma 7 holds in the present context with the change that $u_{i, \max }$ is as in (34). The proof of Lemma 7 is as in Appendix A, except that now, for microgrid $i$, a price of $\tilde{p}$ fetches a payoff of $(\tilde{p}-c)$ if $K_{-i} \geq 1$ and $v \geq \tilde{p}$ and a payoff of 0 otherwise. So $u_{i, \max }=(\tilde{p}-c) P\left(K_{-i} \geq 1\right) P(v \geq \tilde{p})=$ 
$(\tilde{p}-c)\left[1-(1-s)^{n-1}\right]\left(1-G(\tilde{p})\right.$. Thus, $u_{i, \max }$ is given by (34).

Now, in the present context, $\tilde{p}$ is given by Lemma 3 instead of the value in Lemma 9. To prove Lemma 3, we first proceed as in the proof of Lemma 9 described in Appendix A, except that everywhere, we use the fact that if microgrid $i$ sets a price $p_{i}$, then its power is sold only if $v \geq p_{i}$, which results in an additional factor of $\left(1-G\left(p_{i}\right)\right)$ in the expression for microgrid $i$ 's expected payoff. Thus proceeding, we conclude that $\tilde{p}$ must satisfy (33). It remains to show that (33) has a unique solution in $\left(c, v_{T}\right)$. Note that

$$
h(c)=0<\frac{h\left(v_{T}\right)\left(1-w_{1}\right)}{1-(1-s)^{n-1}} .
$$

Let $N_{-i}$ and $K_{-i}$ be as defined in Section III. Clearly, the event $\left\{K_{-1}=0\right\}$ is a strict subset of the event $\left\{N_{-1} \geq K_{-1}\right\}$. So $w_{1}=P\left(N_{-1} \geq K_{-1}\right)>P\left(K_{-1}=0\right)=(1-s)^{n-1}$. Hence:

$$
h\left(v_{T}\right)>\frac{h\left(v_{T}\right)\left(1-w_{1}\right)}{1-(1-s)^{n-1}} .
$$

By Assumption 1, $h($.$) is continuous. So by (90), (91), and$ the intermediate value theorem [6], (33) has a solution in $\left(c, v_{T}\right)$. Also, since $h($.$) is strictly increasing in \left(c, v_{T}\right)$ by Assumption 1, this solution is unique, which completes the proof of Lemma 3.

Finally, Lemmas 12, 11, 10, 13, 14 and 15 go through unchanged and their proofs are similar to those in Appendix A. This completes the proof of the fact that Properties 1 and 2 and Theorem 1 hold with the changes stated in Section V.

Next, we prove Lemma 4 . It can be easily checked using the definition of the function $f_{i}($.$) that f_{i}\left(q_{i}\right) \geq(1-s)^{n-1}$. Hence:

$$
h(\tilde{p}) \leq \frac{h(\tilde{p})\left[1-(1-s)^{n-1}\right]}{1-f_{i}\left(q_{i}\right)}
$$

Also, using the definitions of $f_{i}($.$) and w_{1}$ and equation (1), it can be checked that $f_{i}\left(q_{i}\right) \leq w_{1}$. So:

$$
\begin{aligned}
h\left(v_{T}\right) & \geq \frac{h\left(v_{T}\right)\left(1-w_{1}\right)}{1-f_{i}\left(q_{i}\right)} \\
& =\frac{h(\tilde{p})\left[1-(1-s)^{n-1}\right]}{1-f_{i}\left(q_{i}\right)}
\end{aligned}
$$

where (94) follows from (93) by Lemma 3. By Assumption 1, $h($.$) is continuous. So by (92), (94), and the intermediate value$ theorem [6], (38) has a solution in $\left[\tilde{p}, v_{T}\right]$. Also, since $h($.$) is$ strictly increasing in $\left[\tilde{p}, v_{T}\right]$ by Assumption 1, this solution is unique, which completes the proof of Lemma 4.

Finally, the proof of the fact that Lemma 2 holds in the present context with the changes stated in Section $\mathrm{V}$ is similar to the proof of Lemma 2 in Appendix B. Also, the proof of the fact that Theorem 2 goes through in the present context with the changes stated in Section V is similar to the proof of Theorem 2. 